\title{
Synthesis of 3-(2-aminoethyl)-5-hydroxy-1H-pyrazole derivatives
}

\author{
Uroš Grošelj, ${ }^{a}$ David Kralj, ${ }^{a}$ Jernej Wagger, ${ }^{a}$ Georg Dahmann, ${ }^{b}$ Branko Stanovnik, ${ }^{a}$ \\ and Jurij Svete*a \\ ${ }^{a}$ Faculty of Chemistry and Chemical Technology, University of Ljubljana, Aškerčeva 5, \\ P.O. Box 537, 1000 Ljubljana, Slovenia \\ ${ }^{b}$ Boehringer Ingelheim Pharma GmbH \& Co. KG, Medicinal Chemistry, Birkendorfer Strasse \\ 65, 88397 Biberach, Germany \\ E-mail:jurij.svete@fkkt.uni-lj.si
}

\section{Dedicated to Professor Reiner Beckert, Friedrich Schiller University Jena, on the occasion of his $60^{\text {th }}$ anniversary}

DOI: $\underline{\text { http://dx.doi.org/10.3998/ark.5550190.0013.305 }}$

\begin{abstract}
Treatment of $\beta$-keto ester 8 with hydrazines 9a-g gave 1'-substituted tert-butyl 2-(5-hydroxy-1Hpyrazol-3-yl)ethylcarbamates 10a-e and 2-(5-oxo-2,5-dihydro-1 $H$-pyrazol-3-yl)ethylcarbamates 11f,g. Acidolytic deprotection of 10b,c afforded the corresponding 3-(2-aminoethyl)-5-hydroxy$1 H$-pyrazoles $\mathbf{6 b}, \mathbf{c}$ in good yields. Acylation of $\mathbf{6}$ gave either the $N$-acyl compounds $\mathbf{1 2 b}, \mathbf{c}$ and 13c, or the $N, O$-diacyl derivative 14. Next, three $N, N$-dialkyl analogues $\mathbf{1 5 a}, \mathbf{b}$ and $\mathbf{2 6 c}$ were prepared from dimethyl acetone-1,3-dicarboxylate $\mathbf{2 1}$ via condensation with hydrazines $\mathbf{9 a}$ and $\mathbf{9 h}$ followed by hydrolysis of the esters $\mathbf{2 2 a}, \mathbf{b}$, amidation of the carboxylic acids $\mathbf{2 3 a}, \mathbf{b}$, and reduction of the tertiary carboxamides $\mathbf{2 4 a}$ and $\mathbf{2 5} \mathbf{b}, \mathbf{c}$.
\end{abstract}

Keywords: Acetone-1,3-dicarboxylates, enaminones, hydrazines, pyrazole, histamine analogues

\section{Introduction}

Novel 2-(heteroaryl)ethylamine-containing molecules represent important targets in medicinal and synthetic organic chemistry because these compounds are synthetic analogs of histamine $\mathbf{1}$, tyramine, dopamine, tryptamine, serotonin, and melatonin, which are involved as chemical messengers in numerous biological processes. ${ }^{1}$

Pyrazoles $^{2}$ and imidazoles ${ }^{3}$ are important classes of structurally closely related heterocyclic compounds. The ability of 1-unsubstituted derivatives to act as proton acceptor and donor simultaneously is probably the most important common feature of both systems. In contrast to 
naturally abundant imidazoles, the occurrence of pyrazoles among natural products is rare.

Nevertheless, numerous synthetic pyrazole derivatives found use in various pharmaceutical, agrochemical, and other applications, and a general interest in the chemistry of pyrazoles is still continuing. $^{2}$

Recently, a part of our research has been focused on the synthesis of histamine analogues 2-5 based on aminoethyl functionalized pyrazole scaffold. ${ }^{4-7}$ Within this context, syntheses of analogues $\mathbf{2}^{4}$ and $\mathbf{3}^{5}$ have been developed first, followed by synthesis of conformationally constrained histamine analogues $4^{6}$ and $\mathbf{5}^{7}$. Next, we focused our attention on 3-(2aminoethyl)pyrazol-5-ols 6 as novel type of histamine analogues (Figure 1).

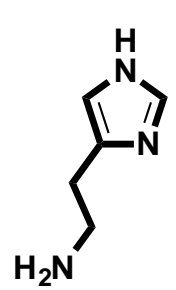

histamine (1)

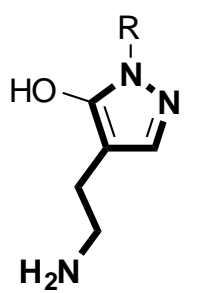

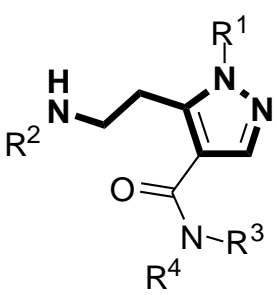

analogue 3

Ref. 5

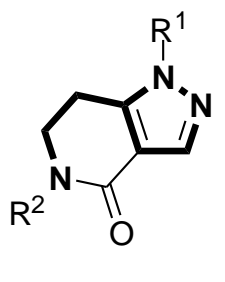

analogue 4 Ref. 6

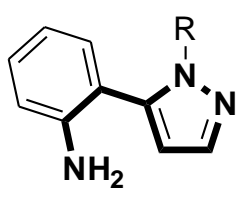

analogue 5

Ref. 7

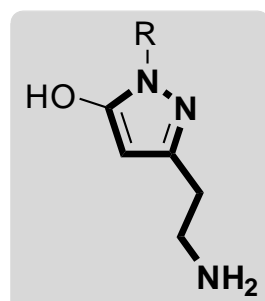

analogue 6

Figure 1. Histamine $\mathbf{1}$ and its analogues 2-6.

We found pyrazolols 6 interesting because they are structurally closer analogues of histamine $\mathbf{1}$ than their known regioisomers 2 . Like histamine 1, compounds 6 have the aminoethyl residue attached at the position adjacent to the ring nitrogen atom. Besides, 3-(2-aminophenyl)pyrazolols could also serve as useful building blocks for further transformations including combinatorial studies. As a result of our research efforts in this field, we now report two simple syntheses of title compounds 6 and their derivatives.

\section{Results and Discussion}

First, a simple and straightforward three-step synthesis of 3-(2-aminoethyl)-1H-pyrazol-5-ols 6 from $N$-Boc- $\beta$-alanine 7 was developed. Following literature procedure, ${ }^{5} \beta$-keto ester $\mathbf{8}$ was prepared from 7 by Masamune-Claisen type condensation. ${ }^{8}$ Further treatment of the $\beta$-keto ester 8 with hydrazine derivatives $9 \mathbf{9}-\mathbf{g}$ in refluxing methanol gave 1'-substituted tert-butyl 2-(5hydroxy-1H-pyrazol-3-yl)ethylcarbamates $\mathbf{1 0 a}-\mathbf{e}$ and 2-(5-oxo-2,5-dihydro-1 $H$-pyrazol-3yl)ethylcarbamates 11f,g in 48-83\% yields. Subsequent acidolytic deprotection of 10b and 10c with $\mathrm{HCl}-\mathrm{EtOAc}$ furnished the title compounds $\mathbf{6 b}$ and $\mathbf{6 c}$ in $78 \%$ and $84 \%$ yield, respectively. Treatment of $\mathbf{6 b}, \mathbf{c}$ with acetic anhydride in methanol produced the $\mathrm{N}$-acetylated compounds $\mathbf{1 2 b}, \mathbf{c}$ in good yields. Similarly, treatment of $\mathbf{6 c}$ with benzoyl chloride in methanol in the presence of triethylamine gave the N-benzoylated compound $\mathbf{1 3 c}$, whereas benzoylation in 
dichloromethane furnished the N,O-dibenzoylated compound $\mathbf{1 4}$ in $79 \%$ yield. O-Benzoylation was not really surprising, since pyrazolones are readily $\mathrm{O}$-acylated with acid chlorides and anhydrides (Scheme 1, Table 1). ${ }^{2}$

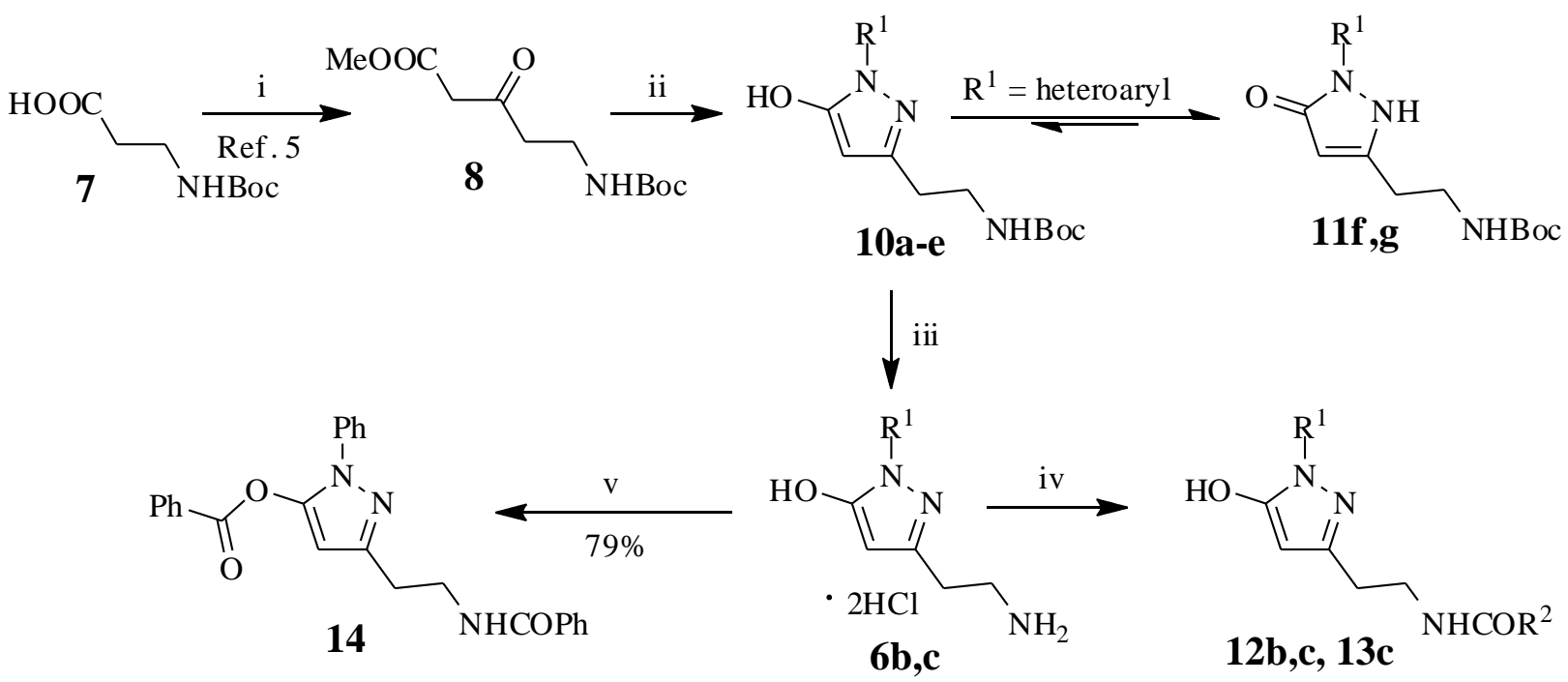

\section{Scheme 1}

Reagents and conditions: i: $\mathrm{CDI}, \mathrm{MeCN}$, r.t., then potassium monomethyl malonate, $\mathrm{MgCl}_{2}$, r.t. ii: $\mathrm{R}^{1} \mathrm{NHNH}_{2}$ 9a-g, $\mathrm{MeOH}$, reflux. iii: $\mathrm{HCl}-\mathrm{EtOAc}$, r.t. iv: $\mathrm{Ac}_{2} \mathrm{O}$ (1 equiv.) or $\mathrm{PhCOCl}$ (1 equiv.), $\mathrm{MeOH}, \mathrm{Et}_{3} \mathrm{~N}, 0 \rightarrow 20^{\circ} \mathrm{C}$. v: $\mathrm{PhCOCl}$ (2 equiv.), $\mathrm{CH}_{2} \mathrm{Cl}_{2}, \mathrm{Et}_{3} \mathrm{~N}$, r.t.

Table 1. Selected experimental data for compounds 6 and 10-13

\begin{tabular}{cccc}
\hline Compound & $\mathrm{R}^{1}$ & $\mathrm{R}^{2}$ & Yield (\%) \\
\hline $\mathbf{6 b}$ & $\mathrm{Me}$ & - & 78 \\
$\mathbf{6 c}$ & $\mathrm{Ph}$ & - & 84 \\
$\mathbf{1 0 a}$ & $\mathrm{H}$ & - & 48 \\
$\mathbf{1 0 b}$ & $\mathrm{Me}$ & - & 73 \\
$\mathbf{1 0 c}$ & $\mathrm{Ph}$ & - & 83 \\
$\mathbf{1 0 d}$ & 4-chlorophenyl & - & 61 \\
$\mathbf{1 0 e}$ & 4-carboxyphenyl & - & 55 \\
$\mathbf{1 1 f}$ & 6-phenylpyridazin-3-yl & - & 81 \\
$\mathbf{1 1 g}$ & imidazo[1,2-b]pyridazin-6-yl & - & 66 \\
$\mathbf{1 2 b}$ & $\mathrm{Me}$ & $\mathrm{Me}$ & 62 \\
$\mathbf{1 2 c}$ & $\mathrm{Ph}$ & $\mathrm{Me}$ & 87 \\
$\mathbf{1 3 c}$ & $\mathrm{Ph}$ & $\mathrm{Ph}$ & 55 \\
\hline
\end{tabular}


Next, we tried to synthesize the $N, N$-dialkyl analogues 15 by a similar synthetic pathway. $N, N$-dialkyl- $\beta$-alanines 19 seemed to be obvious starting materials, since they are available by 1,4-addition of secondary amines $\mathbf{1 7}$ to methyl acrylate $\mathbf{1 6}$ followed by hydrolysis of the esters 18. ${ }^{9}$ Indeed, alaninates $\mathbf{1 8 a ^ { 1 0 a }}$ and $\mathbf{1 8 b ^ { 1 0 b , c }}$ were obtained in quantitative yield by addition of benzyl(methyl)amine $\mathbf{1 7 a}$ and pyrrolidine $\mathbf{1 7 b}$ to methyl acrylate $\mathbf{1 6}$ following a slightly modified literature procedure. ${ }^{10 a}$ Hydrolysis of the esters $\mathbf{1 8 a}, \mathbf{b}$ in aqueous $\mathrm{NaOH}$, followed by neutralization, and isolation by ion-exchange chromatography gave the crude $\beta$-amino acids $\mathbf{1 9} \mathbf{a}^{11 \mathrm{a}}$ and $\mathbf{1 9} \mathbf{b}^{11 \mathrm{~b}}$ in $77 \%$ and $80 \%$ yield, respectively. Unfortunately, all attempts to prepare the $\beta$-keto ester 20 by carboxymethylation of the $\beta$-amino acids 19a,b, either under MasamuneClaisen conditions, or by condensation with Meldrum's acid followed by methanolysis, failed. Nevertheless, this was not surprising, since changing the NHBoc group of compound 7 ( $c f$. Scheme 1) to a strongly basic tertiary amino group of compounds $\mathbf{1 8}$ results in zwitterionic structure and, hence, different reactivity (Scheme 2).

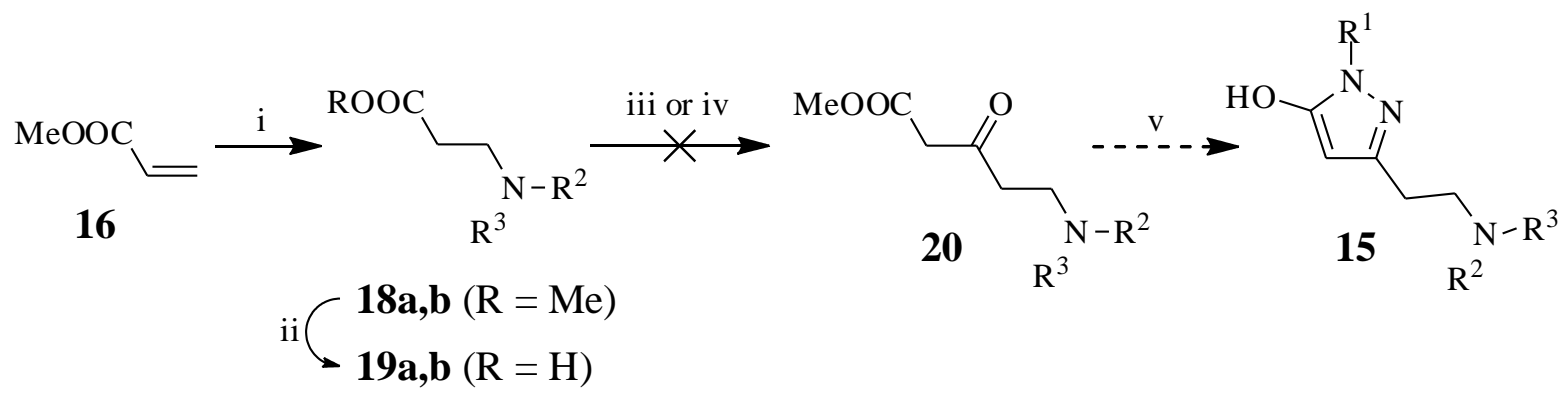

\section{Scheme 2}

Reagents and conditions: i: $\mathrm{Bn}(\mathrm{Me}) \mathrm{NH} \mathbf{1 7 a}$ or pyrrolidine $\mathbf{1 7 b}, \mathrm{CH}_{2} \mathrm{Cl}_{2}$, r.t. ii: $\mathrm{NaOH}, \mathrm{H}_{2} \mathrm{O}$, r.t. iii: $\mathrm{CDI}, \mathrm{MeCN}$, r.t., then potassium monomethyl malonate, $\mathrm{MgCl}_{2}$, r.t. (Method A). iv: Meldrum's acid, DMAP, DCC, THF, r.t., then MeOH, reflux (Method B). v: ${ }^{1} \mathrm{NHNH}_{2} \mathbf{9 c}, \mathbf{h}$, $\mathrm{MeOH}$, reflux.

Our alternative strategy for the preparation of the desired products $\mathbf{1 5}$ started from (pyrazol3-yl)acetates 22, which are easily available from dimethyl acetone-1,3-dicarboxylate $\mathbf{2 1}$ and monosubstituted hydrazines 9.,12 First, methyl (pyrazol-3-yl)acetates 22a,b were prepared from 21 following the literature procedure. ${ }^{12 a}$ Base-catalyzed hydrolysis of $\mathbf{2 2 a}, \mathbf{b}$ gave the acids 23a,b, which were subsequently amidated with the secondary amines $17 \mathbf{a}-\mathbf{c}$ to give the carboxamides $\mathbf{2 4 a}$ and $\mathbf{2 5} \mathbf{b}, \mathbf{c}$ in $42-79 \%$ yields. Reduction of the carboxamides $\mathbf{2 4 a}$ and $\mathbf{2 5 b , c}$ with $\mathrm{LiAlH}_{4}$ in refluxing THF furnished the title compounds 15a,b and 26c in 50-54\% yields (Scheme 3, Table 2). 

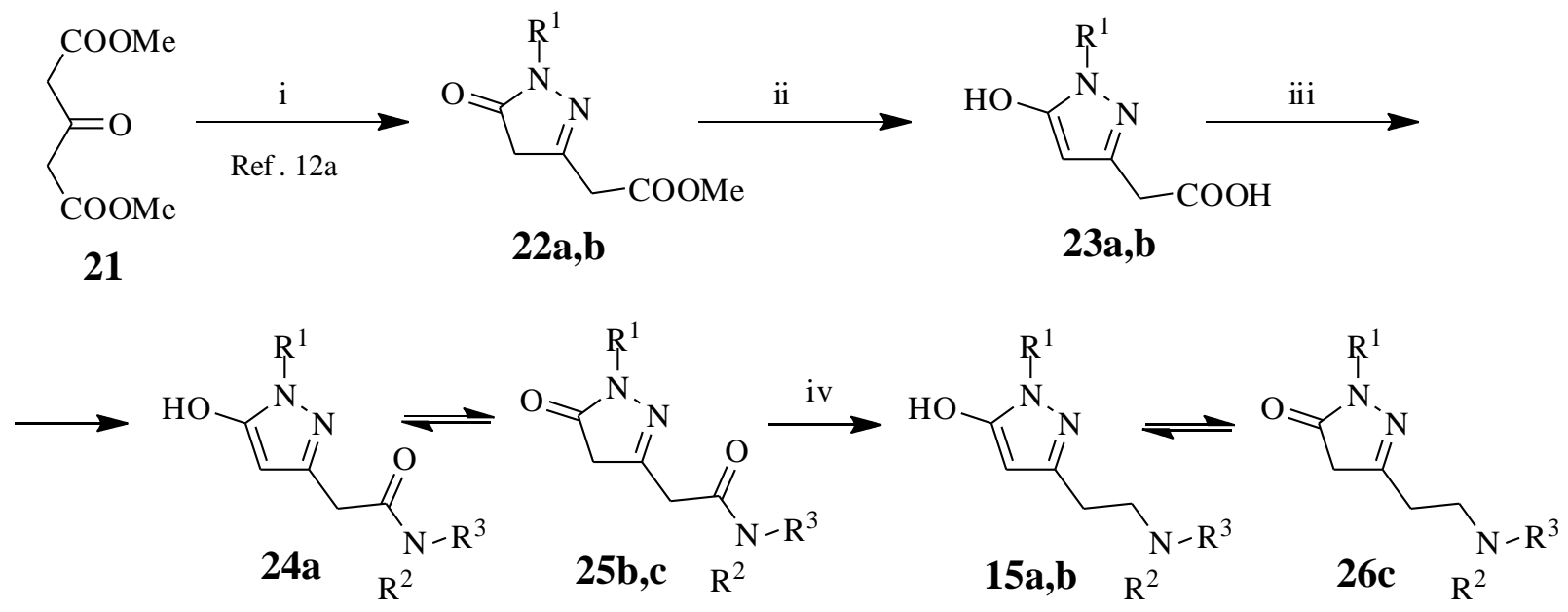

\section{Scheme 3}

Reagents and conditions: i: $\mathrm{R}^{1} \mathrm{NHNH}_{2}$ 9a,h, $\mathrm{MeOH}$, reflux. ${ }^{12 \mathrm{a}}$ ii: $\mathrm{NaOH}-\mathrm{H}_{2} \mathrm{O}$, r.t. iii: CDI, $\mathrm{MeCN}$, r.t., then $\mathrm{Bn}(\mathrm{Me}) \mathrm{NH}$ 17a or pyrrolidine $17 \mathbf{b}$ or $\mathrm{Bn}_{2} \mathrm{NH} 17 \mathbf{c}, \mathrm{MeCN}$, r.t. iv: $\mathrm{LiAlH}_{4}$, THF, $60{ }^{\circ} \mathrm{C}$.

Table 2. Selected experimental data for compounds $\mathbf{1 5}$ and 22-26

\begin{tabular}{|c|c|c|c|c|}
\hline Compound & $\mathrm{R}^{1}$ & $\mathrm{R}^{2}$ & $\mathrm{R}^{3}$ & Yield $(\%)$ \\
\hline $22 \mathbf{a}$ & $\mathrm{Ph}$ & - & - & $62^{12 a}$ \\
\hline $22 b$ & 4-methoxyphenyl & - & - & 43 \\
\hline $23 a$ & $\mathrm{Ph}$ & - & - & 94 \\
\hline $23 b$ & 4-methoxyphenyl & - & - & 79 \\
\hline $24 a$ & $\mathrm{Ph}$ & $\mathrm{Me}$ & $\mathrm{CH}_{2} \mathrm{Ph}$ & 48 \\
\hline $25 b$ & 4-methoxyphenyl & \multicolumn{2}{|c|}{$-\left(\mathrm{CH}_{2}\right)_{4}-$} & 79 \\
\hline $25 c$ & $\mathrm{Ph}$ & $\mathrm{CH}_{2} \mathrm{Ph}$ & $\mathrm{CH}_{2} \mathrm{Ph}$ & 42 \\
\hline $15 \mathbf{a}$ & $\mathrm{Ph}$ & $\mathrm{Me}$ & $\mathrm{CH}_{2} \mathrm{Ph}$ & 50 \\
\hline $15 b$ & 4-methoxyphenyl & \multicolumn{2}{|c|}{$-\left(\mathrm{CH}_{2}\right)_{4-}$} & 54 \\
\hline $26 c$ & $\mathrm{Ph}$ & $\mathrm{CH}_{2} \mathrm{Ph}$ & $\mathrm{CH}_{2} \mathrm{Ph}$ & 50 \\
\hline
\end{tabular}

The structures of novel compounds 6b,c, 10a-e, 11f,g, 12b,c, 13c, 14, 15a,b, 22b, 24a, 25b,c, and 26c were determined by spectroscopic methods (IR, ${ }^{1} \mathrm{H}$ and ${ }^{13} \mathrm{C}$ NMR, NOESY spectroscopy, and MS) and by elemental analyses for $\mathrm{C}, \mathrm{H}$, and N. Compounds 23a,b, 24a, and 25b,c were not obtained in analytically pure form. The identities of carboxamides $\mathbf{2 4 a}$ and $\mathbf{2 5} \mathbf{b}, \mathbf{c}$ were confirmed by ${ }^{1} \mathrm{H}$ NMR, ${ }^{13} \mathrm{C}$ NMR and HRMS, while the intermediate carboxylic acids 23a,b were characterized only by ${ }^{1} \mathrm{H}$ NMR and HRMS. Physical and spectral data for known compounds 18a, ${ }^{10 \mathrm{a}} \mathbf{1 8 b},{ }^{10 \mathrm{~b}, \mathrm{c}} \mathbf{1 9 a},{ }^{11 \mathrm{a}} \mathbf{1 9 b},{ }^{1 \mathrm{~b}}$ and $22 \mathbf{a}^{12 \mathrm{a}}$ were in agreement with the literature data. 
Like related 5-hydroxypyrazoles, ${ }^{2-4,13}$ the novel derivatives 6, 10-13, 15, and 22-26 can exist in three tautomeric forms, the fully unsaturated "OH-tautomer" (5-hydroxy-1H-pyrazole, 6, 10, 12-15, 23, 24) and the partially unsaturated "NH-tautomer" $(1 H$-pyrazol-3(2H)-one, 11) and "CH-tautomer" (1H-pyrazol-5(4H)-one, 22, 25, 26). In the solid state, the tautomerism of novel compounds 6, 10-13, 15, and 22-26 was studied by IR. Absence of $\mathrm{C}=\mathrm{O}$ vibrations indicate that pyrazoles 6b,c and 15a,b exist as the 5-hydroxy-1H-pyrazoles (OH-tautomers), whereas absorption at $1700-1750 \mathrm{~cm}^{-1}$ is in agreement with the $1 H$-pyrazol-5(4H)-one form for compounds 22b, 25b,c, and 26c (CH-tautomers). Unambiguous discrimination between the $\mathrm{OH}-$ and the NH-tautomers for the $N$-acylated compounds 10-13 and 24 was not possible, due to carboxamide absorption at $\sim 1640 \mathrm{~cm}^{-1}$. However, the absorption band at $\sim 1690 \mathrm{~cm}^{-1}$ (Boc) and absence of $\mathrm{C}=\mathrm{O}$ vibrations at $\sim 1640 \mathrm{~cm}^{-1}$ support the $\mathrm{OH}$-tautomeric form of $\mathbf{1 0 a}-\mathbf{e}$, while absorption bands at $\sim 1690 \mathrm{~cm}^{-1}$ and $\sim 1640 \mathrm{~cm}^{-1}$ are in agreement with the NH-tautomeric form of 1-heteroarylpyrazoles 11f,g. Accordingly, the $N$-acylated 1-methylpyrazole $\mathbf{1 2 b}$ and 1phenylpyrazoles 12c, 13c, and 24a exhibiting single $\mathrm{C}=\mathrm{O}$ absorption bands at $\sim 1640 \mathrm{~cm}^{-1}$ most probably exist as the $\mathrm{OH}$-tautomers. In solution, the tautomer equilibrium was solvent-depended. In DMSO- $d_{6}$, chemical shifts of 4-H $(\delta \sim 5.5 \mathrm{ppm})$ and $5-\mathrm{C}(\delta \sim 153 \mathrm{ppm})$ were in agreement with the $1 H$-pyrazol-5-ols 6b,c, 10a-e, 12b,c, 13c, 15b, 22b, 23a,b, 24a, 25b,c, and 26c. Broad signals for the 4-H and methylene protons and the corresponding carbon nuclei indicated fast tautomerisation between the $\mathrm{OH}$ - and the $\mathrm{NH}$-tautomer in these compounds. In $\mathrm{CDCl}_{3}$, on the other hand, a singlet for the $4-\mathrm{CH}_{2}$ group at $\sim 3.5 \mathrm{ppm}$ clearly indicated the $1 H$-pyrazol-5(4H)ones 13c, 15a, 22b, 24a, 25b,c, and 26c. These data are also in agreement with the literature data on tautomerism of related pyrazolones (Figure 2).,2,13

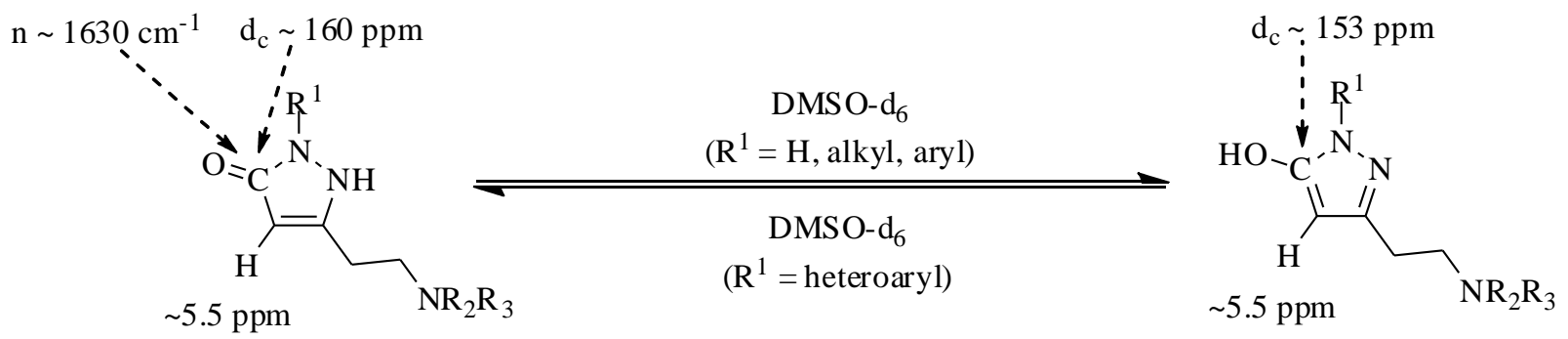

11f,g

(NH-tautomers)
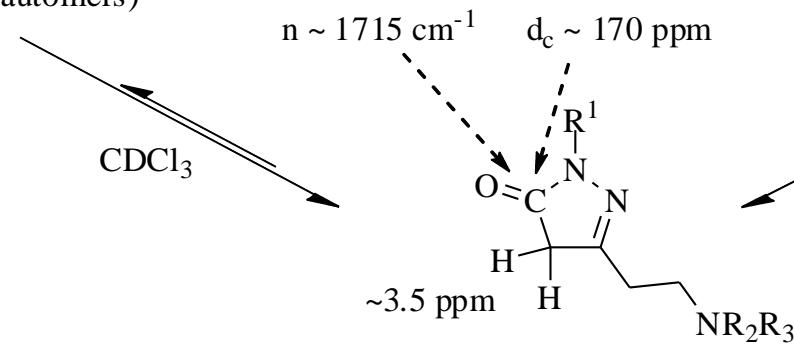

6b,c, 10a-e, 12b,c, 13c, 15b,

22b, 23a,b, 24a, 25b,c, 26c

(OH-tautomers)

13c, 15a, 22b, 24a, 25b,c, 26c

(CH-tautomers)

Figure 2. Tautomeric forms of pyrazole derivatives 6, 10-13, 15, and 22-26. 


\section{Conclusions}

In summary, two synthetic methods for the preparation of a novel type of pyrazole analogues of histamine 6, 15, and 26 were developed. The first method starts from Boc- $\beta$-alanine 7, which is transformed in three steps into the title compounds, 1-substituted 3-(2-aminophenyl)-1H-pyrazol$5(4 H)$-ones 6. Further acylation of $\mathbf{6}$ in methanol produced the $N$-acyl derivatives $\mathbf{1 2}$ and 13, while acylation of $\mathbf{6 c}$ in dichloromethane led to the N,O-diacylated compound 14. The second method enables access to $N, N$-dialkyl analogues 15. It comprises cyclisation of dialkyl acetone-1,3dicarboxylate $\mathbf{2 1}$ with monosubstituted hydrazines 9 to give alkyl pyrazolone-3-acetates $\mathbf{2 2}$, followed by a three-step transformation into 3-(2-(dialkylamino)phenyl)- $1 H$-pyrazol-5-ols $\mathbf{1 5}$ and 26. These synthetic methods enable easy access to a novel type of histamine analogues as interesting molecules for biological studies.

\section{Experimental Section}

General. Melting points were determined on a Kofler micro hot stage and on a Stanford Research Systems MPA100 OptiMelt automated melting point system. The NMR spectra were obtained on a Bruker Avance DPX 300 at $300 \mathrm{MHz}$ for ${ }^{1} \mathrm{H}$ and $75.5 \mathrm{MHz}$ for ${ }^{13} \mathrm{C}$ nucleus and on Bruker Avance III UltraShield 500 plus at $500 \mathrm{MHz}$ for ${ }^{1} \mathrm{H}$ and $126 \mathrm{MHz}$ for ${ }^{13} \mathrm{C}$ nucleus, using DMSO- $d_{6}$ and $\mathrm{CDCl}_{3}$ with TMS as the internal standard, as solvents. Mass spectra were recorded on an AutoSpecQ spectrometer and Agilent 6224 Accurate Mass TOF LC/MS, IR spectra on a Perkin-Elmer Spectrum BX FTIR spectrophotometer. Microanalyses were performed on a Perkin-Elmer CHN Analyzer 2400 II. Column chromatography (CC) and flash chromatography (FC) were performed on silica gel (Fluka, Silica gel 60, particle size: 0.035-0.070 mm).

Boc- $\beta$-alanine 7, hydrazines 9a-h, methyl acrylate 16, amines 17a-c, and dimethyl acetone-1,3dicarboxylate $\mathbf{2 1}$ are commercially available (Sigma-Aldrich). Methyl 5-(tertbutoxycarbonylamino)-3-oxopentanoate $\mathbf{8}^{5}$ and methyl 2-(5-oxo-1-phenyl-4,5-dihydro- $1 \mathrm{H}$ pyrazol-3-yl)acetate $2 \mathbf{a}^{12 \mathrm{a}}$ were prepared following the literature procedures.

\section{Preparation of 1'-substituted tert-butyl 2-(5-hydroxy-1H-pyrazol-3-yl)ethylcarbamates 10a-e and tert-butyl 2-(5-oxo-2,5-dihydro-1H-pyrazol-3-yl)ethylcarbamates 11f,g. A mixture of $8(245 \mathrm{mg}, 1 \mathrm{mmol})$, methanol $(5 \mathrm{~mL})$, and hydrazine derivative $9 \mathbf{9}-\mathbf{g}(1 \mathrm{mmol})^{14}$ was stirred under reflux for $5 \mathrm{~h}$, and cooled to r.t. Compounds 10a, 10e, and 11f precipitated from the reaction mixtures and were collected by filtration to give 10a,e and 11f. Compounds 10b-d and $11 \mathrm{~g}$ did not precipitate from the reaction mixtures, which were evaporated in vacuo and the residues were chromatographed over silica gel (EtOH/EtOAc or EtOAc/hexanes, column dimensions: $1.5 \times 7 \mathrm{~cm}$ ). Fractions containing the product were combined and evaporated in vacuo to give $\mathbf{1 0 b - d}$ and $\mathbf{1 1 g}$.}


tert-Butyl 2-(5-hydroxy-1H-pyrazol-3-yl)ethylcarbamate (10a). Prepared from 8 (245 mg, 1 mmol) and hydrazine hydrate $9 \mathbf{a}(50 \mu \mathrm{L}, 50 \mathrm{mg}, 1 \mathrm{mmol})$. White solid, yield 48\%, $108 \mathrm{mg}, \mathrm{mp}$ 188-191 ${ }^{\circ} \mathrm{C}$, IR ( $\left.v_{\max }, \mathrm{cm}^{-1}\right): 3380,2982,1689(\mathrm{C}=\mathrm{O}), 1613,1528,1460,1364,1271,1246$, 1171, 974, 759. ${ }^{1} \mathrm{H}$ NMR $\left(500 \mathrm{MHz}, \mathrm{DMSO}-d_{6}\right), \delta_{\mathrm{H}} 1.38(9 \mathrm{H}, \mathrm{s}, t-\mathrm{Bu}), 2.56\left(2 \mathrm{H}, \mathrm{t},{ }^{3} J_{\mathrm{HH}}=7.5\right.$ $\left.\mathrm{Hz}, \mathrm{CH}_{2} \mathrm{CH}_{2} \mathrm{NH}\right), 3.10\left(2 \mathrm{H}\right.$, br q, $\left.{ }^{3} \mathrm{~J}_{\mathrm{HH}}=6.9 \mathrm{~Hz}, \mathrm{CH}_{2} \mathrm{CH}_{2} \mathrm{NH}\right), 5.25(1 \mathrm{H}$, br s, 4-H of pyrazole), $6.86\left(1 \mathrm{H}\right.$, br t, $\left.{ }^{3} J_{\mathrm{HH}}=6.3 \mathrm{~Hz}, \mathrm{NHBoc}\right), 9.44(1 \mathrm{H}$, br s, $1-\mathrm{H}), 11.14(1 \mathrm{H}, \mathrm{br} \mathrm{s}, \mathrm{OH}) .{ }^{13} \mathrm{C} \mathrm{NMR}(126$ MHz, DMSO- $\left.d_{6}\right), \delta_{\mathrm{C}} 26.5,28.3,77.6,77.8,88.3,141.8,155.5,160.8 . \mathrm{MS}, m / z=228\left(\mathrm{MH}^{+}\right)$, HRMS (ESI), $m / z=228.1335\left(\mathrm{MH}^{+}\right), \mathrm{C}_{10} \mathrm{H}_{18} \mathrm{~N}_{3} \mathrm{O}_{3}$ requires 228.1343. Anal. Calcd for $\mathrm{C}_{10} \mathrm{H}_{17} \mathrm{~N}_{3} \mathrm{O}_{3}$ (227.26): C, 52.85; H, 7.84; N, 18.49\%, Found: C, 52.83; H, 7.60; N; $18.46 \%$.

tert-Butyl 2-(5-hydroxy-1-methyl-1H-pyrazol-3-yl)ethylcarbamate (10b). Prepared from 8 (245 mg, $1 \mathrm{mmol}$ ) and methylhydrazine 9b (50 $\mu \mathrm{L}, 46 \mathrm{mg}, 1 \mathrm{mmol})$, CC (EtOAc/hexanes, 1:1). White solid, yield 73\%, $177 \mathrm{mg}, \mathrm{mp} 160-162{ }^{\circ} \mathrm{C}$, IR $\left(v_{\max }, \mathrm{cm}^{-1}\right): 3376,2982,1690(\mathrm{C}=\mathrm{O})$, $1533,1459,1401,1270,1173,1039,1000,974,748,684 .{ }^{1} \mathrm{H}$ NMR (300 MHz, DMSO- $\left.d_{6}\right), \delta_{\mathrm{H}}$ $1.37(9 \mathrm{H}, \mathrm{s}, t-\mathrm{Bu}), 2.45\left(2 \mathrm{H}\right.$, bt t, $\left.{ }^{3} \mathrm{~J}_{\mathrm{HH}}=7.7 \mathrm{~Hz}, \mathrm{CH}_{2} \mathrm{CH}_{2} \mathrm{NH}\right), 3.08\left(2 \mathrm{H}, \mathrm{br} \mathrm{m}, \mathrm{CH}_{2} \mathrm{CH}_{2} \mathrm{NH}\right), 3.41$ (3H, s, NMe), 5.16 (1H, br s, 4-H of pyrazole), 6.73 (1H, br s, NHBoc), 10.64 (1H, br s, OH). ${ }^{13} \mathrm{C}$ NMR $\left(75.5 \mathrm{MHz}\right.$, DMSO- $\left.d_{6}\right)$, $\delta_{\mathrm{C}} 28.3,29.1,32.5,77.5,77.8,85.2$, 147.3, 153.0, 155.5. MS, $m / z=242\left(\mathrm{MH}^{+}\right)$, HRMS $(\mathrm{ESI}), m / z=242.1494\left(\mathrm{MH}^{+}\right), \mathrm{C}_{11} \mathrm{H}_{19} \mathrm{~N}_{3} \mathrm{O}_{3}$ requires 242.1499. Anal. Calcd for $\mathrm{C}_{11} \mathrm{H}_{19} \mathrm{~N}_{3} \mathrm{O}_{3}$ (241.29): C, 54.76; H, 7.94; N, 17.41\%, Found: C, 54.76; H, 8.05; N; $17.29 \%$.

tert-Butyl 2-(5-hydroxy-1-phenyl-1H-pyrazol-3-yl)ethylcarbamate (10c). Prepared from 8 (245 mg, $1 \mathrm{mmol})$ and phenylhydrazine 9c $(103 \mu \mathrm{L}, 108 \mathrm{mg}, 1 \mathrm{mmol}), \mathrm{CC}$ (EtOAc/hexanes, 1:2). Beige solid, yield 83\%, $250 \mathrm{mg}, \mathrm{mp} 155-157^{\circ} \mathrm{C}$. IR $\left(v_{\max }, \mathrm{cm}^{-1}\right): 3218,3050,2866,1669$ (C=O), 1601, 1560, 1455, 1409, 1365, 1306, 1256, 1160, 1060, 1037, 964, 868, 761, 694, 644. ${ }^{1} \mathrm{H}$ NMR $\left(300 \mathrm{MHz}, \mathrm{CDCl}_{3}\right), \delta_{\mathrm{H}} 1.41(9 \mathrm{H}, \mathrm{s}, t-\mathrm{Bu}), 2.67\left(2 \mathrm{H}, \mathrm{br} \mathrm{t},{ }^{3} J_{\mathrm{HH}}=6.3 \mathrm{~Hz}, \mathrm{CH}_{2} \mathrm{CH}_{2} \mathrm{NH}\right)$, $3.49\left(2 \mathrm{H}\right.$, br s, 4'- $\left.\mathrm{CH}_{2}\right), 3.52\left(2 \mathrm{H}\right.$, br q, $\left.{ }^{3} J_{\mathrm{HH}}=6.2 \mathrm{~Hz}, \mathrm{CH}_{2} \mathrm{CH}_{2} \mathrm{NH}\right), 4.87(1 \mathrm{H}$, br s, NHBoc), $7.19\left(1 \mathrm{H}, \mathrm{tt},{ }^{4} J_{\mathrm{HH}}=1.1 \mathrm{~Hz},{ }^{3} J_{\mathrm{HH}}=7.4 \mathrm{~Hz}, p-\mathrm{Ph}\right), 7.36-7.40(2 \mathrm{H}, \mathrm{m}, m-\mathrm{Ph}), 7.82-7.88(2 \mathrm{H}, \mathrm{m}, o-$ $\mathrm{Ph}) .{ }^{1} \mathrm{H}$ NMR $\left(500 \mathrm{MHz}, \mathrm{DMSO}-d_{6}\right), \delta_{\mathrm{H}} 1.38(9 \mathrm{H}, \mathrm{s}, t-\mathrm{Bu}), 2.58\left(2 \mathrm{H}\right.$, br t, ${ }^{3} J_{\mathrm{HH}}=7.5 \mathrm{~Hz}$, $\left.\mathrm{CH}_{2} \mathrm{CH}_{2} \mathrm{NH}\right), 3.18\left(2 \mathrm{H}\right.$, br q, $\left.{ }^{3} J_{\mathrm{HH}}=6.8 \mathrm{~Hz}, \mathrm{CH}_{2} \mathrm{CH}_{2} \mathrm{NH}\right), 5.40(1 \mathrm{H}$, br s, 4-H of pyrazole), 6.88 $\left(1 \mathrm{H}\right.$, br s, NHBoc), $7.21\left(1 \mathrm{H}, \mathrm{t},{ }^{3} J_{\mathrm{HH}}=7.4 \mathrm{~Hz}, p-\mathrm{Ph}\right), 7.41\left(2 \mathrm{H}, \mathrm{t},{ }^{3} J_{\mathrm{HH}}=7.9 \mathrm{~Hz}, m-\mathrm{Ph}\right), 7.68(2 \mathrm{H}$, $\left.\mathrm{d},{ }^{3} J_{\mathrm{HH}}=7.8 \mathrm{~Hz}, o-\mathrm{Ph}\right), 11.53\left(1 \mathrm{H}\right.$, br s, OH). ${ }^{13} \mathrm{C}$ NMR $\left(126 \mathrm{MHz}, \mathrm{DMSO}-d_{6}\right), \delta_{\mathrm{C}} 28.2,28.3$, 29.2 , 77.6, 87.0, 120.6, 125.1, 128.8, 138.9, 150.0, 153.1, 155.5. MS, $m / z=304\left(\mathrm{MH}^{+}\right)$, HRMS (ESI), $m / z=304.1650\left(\mathrm{MH}^{+}\right), \mathrm{C}_{16} \mathrm{H}_{22} \mathrm{~N}_{3} \mathrm{O}_{3}$ requires 304.1656. Anal. Calcd for $\mathrm{C}_{16} \mathrm{H}_{21} \mathrm{~N}_{3} \mathrm{O}_{3}$ (303.36): C, 63.35; H, 6.98; N, 13.85\%, Found: C, 63.24; H, 7.08; N, $13.60 \%$.

tert-Butyl 2-(5-hydroxy-1-(4-chlorophenyl)-1H-pyrazol-3-yl)ethylcarbamate (10d). Prepared from 8 (245 mg, $1 \mathrm{mmol}$ ) and 4-chlorophenylhydrazine hydrochloride 9d (179 mg, $1 \mathrm{mmol}), \mathrm{CC}$ (EtOAc/hexanes, 1:2). Grayish solid, yield 61\%, $206 \mathrm{mg}, \mathrm{mp} 107-108{ }^{\circ} \mathrm{C}$. IR $\left(v_{\max }, \mathrm{cm}^{-1}\right): 3393$, 2980, 2928, 1688 (C=O), 1526, 1493, 1397, 1366, 1274, 1254, 1168, 1092, 1028, 1012, 842, 788, 755, 659. ${ }^{1} \mathrm{H}$ NMR $\left(300 \mathrm{MHz}, \mathrm{CDCl}_{3}\right), \delta_{\mathrm{H}} 1.39(9 \mathrm{H}, \mathrm{s}, t-\mathrm{Bu}), 2.66\left(2 \mathrm{H}, \mathrm{br} \mathrm{t},{ }^{3} J_{\mathrm{HH}}=6.3 \mathrm{~Hz}\right.$, $\left.\mathrm{CH}_{2} \mathrm{CH}_{2} \mathrm{NH}\right), 3.49\left(2 \mathrm{H}\right.$, br s, 4'- $\left.\mathrm{CH}_{2}\right), 3.49\left(2 \mathrm{H}\right.$, br q, $\left.{ }^{3} \mathrm{~J}_{\mathrm{HH}}=6.3 \mathrm{~Hz}, \mathrm{CH}_{2} \mathrm{CH}_{2} \mathrm{NH}\right), 4.83(1 \mathrm{H}$, br s, $\mathrm{NHBoc}), 7.34$ and $7.83\left(4 \mathrm{H}, 2 \mathrm{dt}, 1: 1,{ }^{3} J_{\mathrm{HH}}=2.6,9.0 \mathrm{~Hz}, \mathrm{C}_{6} \mathrm{H}_{4}\right) .{ }^{1} \mathrm{H}$ NMR $\left(500 \mathrm{MHz}\right.$, DMSO- $\left.d_{6}\right)$, 
$\delta_{\mathrm{H}} 1.38(9 \mathrm{H}, \mathrm{s}, t-\mathrm{Bu}), 2.58\left(2 \mathrm{H}, \mathrm{br} \mathrm{t},{ }^{3} J_{\mathrm{HH}}=7.2 \mathrm{~Hz}, \mathrm{CH}_{2} \mathrm{CH}_{2} \mathrm{NH}\right), 3.18\left(2 \mathrm{H}, \mathrm{br} \mathrm{q},{ }^{3} J_{\mathrm{HH}}=6.5 \mathrm{~Hz}\right.$, $\left.\mathrm{CH}_{2} \mathrm{CH}_{2} \mathrm{NH}\right), 5.42(1 \mathrm{H}, \mathrm{s}, 4-\mathrm{H}$ of pyrazole), 6.88 (1H, br s, NHBoc), 7.48 and 7.77 (4H, 2dt, 1:1, $\left.{ }^{3} J_{\mathrm{HH}}=2,5,8.7 \mathrm{~Hz}, \mathrm{C}_{6} \mathrm{H}_{4}\right), 11.73\left(1 \mathrm{H}\right.$, br s, OH). ${ }^{13} \mathrm{C}$ NMR (126 MHz, DMSO- $\left.d_{6}\right), \delta_{\mathrm{C}} 28.1,28.3$, 29.2 , 77.5, 87.1, 121.8, 128.8, 129.0, 137.9, 150.4, 153.2, 155.5. MS, $m / z=338\left(\mathrm{MH}^{+}\right)$, HRMS (ESI), $m / z=338.1264\left(\mathrm{MH}^{+}\right), \mathrm{C}_{16} \mathrm{H}_{21} \mathrm{ClN}_{3} \mathrm{O}_{3}$ requires 338.1266. Anal. Calcd for $\mathrm{C}_{16} \mathrm{H}_{20} \mathrm{ClN}_{3} \mathrm{O}_{3}$ (337.80): C, 56.89; H, 5.97; N, 12.44\%, Found: C, 56.87; H, 5.80; N, $12.28 \%$.

4-(3-(2-(tert-butoxycarbonylamino)ethyl)-5-hydroxy-1H-pyrazol-1-yl)benzoic acid (10e). Prepared from 8 (245 mg, $1 \mathrm{mmol})$ and 4-hydrazinobenzoic acid 9e (152 $\mathrm{mg}, 1 \mathrm{mmol})$. White solid, yield 55\%, $192 \mathrm{mg}, \mathrm{mp} 187-189{ }^{\circ} \mathrm{C}$ (decomp.), IR ( $\left.v_{\max }, \mathrm{cm}^{-1}\right): 3328,1691$ (C=O), 1649 $(\mathrm{C}=\mathrm{O}), 1621,1604,1588,1573,1539,1511,1407,1366,1337,1237,1186,1154,993,854,773$, 703, 684. ${ }^{1} \mathrm{H}$ NMR (300 MHz, DMSO- $\left.d_{6}\right), \delta_{\mathrm{H}} 1.38(9 \mathrm{H}, \mathrm{s}, t-\mathrm{Bu}), 2.60\left(2 \mathrm{H}, \mathrm{br} \mathrm{t},{ }^{3} J_{\mathrm{HH}}=7.3 \mathrm{~Hz}\right.$, $\left.\mathrm{CH}_{2} \mathrm{CH}_{2} \mathrm{NH}\right), 3.19\left(2 \mathrm{H}\right.$, br q, $\left.{ }^{3} J_{\mathrm{HH}}=6.7 \mathrm{~Hz}, \mathrm{CH}_{2} \mathrm{CH}_{2} \mathrm{NH}\right), 5.46(1 \mathrm{H}$, br s, 4-H of pyrazole), 6.89 (1H, br s, NHBoc), 7.91 and $7.98\left(4 \mathrm{H}, 2 \mathrm{br} \mathrm{d}, 1: 1,{ }^{3} \mathrm{~J}_{\mathrm{HH}}=8.7 \mathrm{~Hz}, \mathrm{C}_{6} \mathrm{H}_{4}\right), 11.90$ and 12.89 (2H, 2 br s, 1:1, OH and $\mathrm{COOH}) .{ }^{13} \mathrm{C}$ NMR (75.5 MHz, DMSO-d6), $\delta_{\mathrm{C}} 28.1,28.3,29.3,77.6,87.5$, $119.4,126.7,130.3,142.5,151.1,153.8,155.5,166.9 . \mathrm{MS}, m / z=348\left(\mathrm{MH}^{+}\right), \mathrm{HRMS}(\mathrm{ESI}), \mathrm{m} / z$ $=348.1548\left(\mathrm{MH}^{+}\right), \mathrm{C}_{17} \mathrm{H}_{22} \mathrm{~N}_{3} \mathrm{O}_{5}$ requires 348.1554. Anal. Calcd for $\mathrm{C}_{17} \mathrm{H}_{21} \mathrm{~N}_{3} \mathrm{O}_{5}$ (347.37): C, 58.78; H, 6.09; N, 12.10\%, Found: C, 58.78; H, 6.16; N, 12.06\%.

tert-Butyl 2-(5-oxo-1-(6-phenylpyridazin-3-yl)-2,5-dihydro- $\boldsymbol{H}$-pyrazol-3-yl)ethylcarbamate (11f). Prepared from 8 (245 mg, $1 \mathrm{mmol})$ and 3-hydrazino-6-phenylpyridazine 9f (186 mg, 1 mmol). White solid, yield $81 \%, 310 \mathrm{mg}, \mathrm{mp} 242-246{ }^{\circ} \mathrm{C}, \mathrm{IR}\left(v_{\max }, \mathrm{cm}^{-1}\right): 3321,3076,1713$ $(\mathrm{C}=\mathrm{O}), 1634$ (C=O), 1562, 1547, 1455, 1422, 1366, 1296, 1247, 1167, 1131, 784, 690. ${ }^{1} \mathrm{H}$ NMR $\left(300 \mathrm{MHz}, \mathrm{DMSO}-d_{6}\right), \delta_{\mathrm{H}} 1.38(9 \mathrm{H}, \mathrm{s}, t-\mathrm{Bu}), 2.69\left(2 \mathrm{H}\right.$, br t, $\left.{ }^{3} \mathrm{~J}_{\mathrm{HH}}=7.0 \mathrm{~Hz}, \mathrm{CH}_{2} \mathrm{CH}_{2} \mathrm{NH}\right), 3.25$ $\left(2 \mathrm{H}\right.$, br q, $\left.{ }^{3} J_{\mathrm{HH}}=6.8 \mathrm{~Hz}, \mathrm{CH}_{2} \mathrm{CH}_{2} \mathrm{NH}\right), 5.28(1 \mathrm{H}$, br s, $4-\mathrm{H}$ of pyrazole $), 6.97\left(1 \mathrm{H}, \mathrm{br} \mathrm{t},{ }^{3} J_{\mathrm{HH}}=5.0\right.$ $\mathrm{Hz}, \mathrm{NHBoc}), 7.49-7.61(3 \mathrm{H}, \mathrm{m}, p-\mathrm{Ph}, m-\mathrm{Ph}), 8.12-8.17(2 \mathrm{H}, \mathrm{m}, o-\mathrm{Ph}), 8.36\left(1 \mathrm{H}, \mathrm{d},{ }^{3} J_{\mathrm{HH}}=9.4\right.$ $\mathrm{Hz}, 4 "-\mathrm{H}), 8.65\left(1 \mathrm{H}\right.$, br s, 5"-H), $12.52\left(1 \mathrm{H}\right.$, br s, 2'-H). ${ }^{13} \mathrm{C}$ NMR (126 MHz, DMSO- $\left.d_{6}\right), \delta_{\mathrm{C}}$ 26.8, 28.3, 38.4, 77.7, 101.7, 116.7, 126.1, 126.4, 129.1, 129.9, 135.6, 153.3, 155.5, 162.7, 167.0, 171.4. MS, $m / z=382\left(\mathrm{MH}^{+}\right)$, HRMS (ESI), $m / z=382.1866\left(\mathrm{MH}^{+}\right), \mathrm{C}_{20} \mathrm{H}_{24} \mathrm{~N}_{5} \mathrm{O}_{3}$ requires 382.1874. Anal. Calcd for $\mathrm{C}_{20} \mathrm{H}_{23} \mathrm{~N}_{5} \mathrm{O}_{3}$ (381.43): C, 62.98; H, 6.08; N, 18.36\%, Found: C, 62.85; H, 5.99; N; 18.46\%.

tert-Butyl 2-(1-(imidazo[1,2-b]pyridazin-6-yl)-5-oxo-2,5-dihydro-1H-pyrazol-3-yl)ethylcarbamate (11g). Prepared from $8(245 \mathrm{mg}, 1 \mathrm{mmol})$ and 6-hydrazinoimidazo[4,3- $b$ ]pyridazine 9g (149 mg, $1 \mathrm{mmol}), \mathrm{CC}$ : EtOAc. Yellowish solid, yield 66\%, $227 \mathrm{mg}, \mathrm{mp} \mathrm{221-225}{ }^{\circ} \mathrm{C}$, IR $\left(v_{\max }, \mathrm{cm}^{-1}\right): 3370,3137,2978,1716(\mathrm{C}=\mathrm{O}), 1684(\mathrm{C}=\mathrm{O}), 1638(\mathrm{C}=\mathrm{O}), 1573,1544,1522,1403$, 1403, 1366, 1327, 1288, 1250, 1168, 1061, 813, 778. ${ }^{1} \mathrm{H}$ NMR (300 MHz, DMSO-d6), $\delta_{\mathrm{H}} 1.38$ $(9 \mathrm{H}, \mathrm{s}, t-\mathrm{Bu}), 2.64\left(2 \mathrm{H}\right.$, br t, $\left.{ }^{3} J_{\mathrm{HH}}=7.1 \mathrm{~Hz}, \mathrm{CH}_{2} \mathrm{CH}_{2} \mathrm{NH}\right), 3.23\left(2 \mathrm{H}\right.$, br q, ${ }^{3} J_{\mathrm{HH}}=7.1 \mathrm{~Hz}$, $\left.\mathrm{CH}_{2} \mathrm{CH}_{2} \mathrm{NH}\right), 5.28$ (1H, br s, 4-H of pyrazole), 6.94 (1H, br s, NHBoc), 7.76-7.78 (1H, m, 3"-H), 8.15-8.28 (3H, m, 2"-H, 7"-H, 8"-H), 11.92 (1H, br s, 2'-H). ${ }^{13} \mathrm{C}$ NMR (126 MHz, DMSO- $\left.d_{6}\right), \delta_{\mathrm{C}}$ 28.1, 28.3, 38.7, 77.7, 88.0, 92.4, 110.2, 117.2, 127.1, 133.7, 137.1, 146.4, 153.9, 155.6. MS, $\mathrm{m} / z$ $=345\left(\mathrm{MH}^{+}\right)$, HRMS (ESI), $m / z=345.1669\left(\mathrm{MH}^{+}\right), \mathrm{C}_{16} \mathrm{H}_{21} \mathrm{~N}_{6} \mathrm{O}_{3}$ requires 345.1670. Anal. Calcd for $\mathrm{C}_{16} \mathrm{H}_{20} \mathrm{~N}_{6} \mathrm{O}_{3}$ (344.37): C, 55.80; H, 5.85; N, 24.40\%, Found: C, 55.73; H, 5.87; N; $24.33 \%$. 
General procedure for the synthesis of 1-substituted 3-(2-aminoethyl)-5-hydroxy-1Hpyrazoles dihydrochlorides $(6 \mathrm{~b}, \mathrm{c})$

$2 \mathrm{M} \mathrm{HCl}-E t O A c$ ( $25 \mathrm{~mL}, 50 \mathrm{mmol})$ was added to a stirred suspension of $\mathbf{1 0}(5 \mathrm{mmol})$ in anhydrous ethanol $(25 \mathrm{~mL})$ and the mixture was stirred at r.t. for $3 \mathrm{~h}$. The precipitate was collected by filtration and washed subsequently with EtOAc $(25 \mathrm{~mL})$ and ether $(25 \mathrm{~mL})$ to give 6.

3-(2-Aminoethyl)-5-hydroxy-1-methyl-1H-pyrazole dihydrochloride (6b). Prepared from $10 b$ (1.206 g, $5 \mathrm{mmol})$. White solid, yield 78\%, $832 \mathrm{mg}, \mathrm{mp} 197-200{ }^{\circ} \mathrm{C}$, IR ( $\left.v_{\max }, \mathrm{cm}^{-1}\right): 1609$, $1539,1287,1096,945,831,673 .{ }^{1} \mathrm{H}$ NMR (500 MHz, DMSO- $\left.d_{6}\right), \delta_{\mathrm{H}} 2.90\left(2 \mathrm{H}\right.$, br t, ${ }^{3} J_{\mathrm{HH}}=7.2$ $\left.\mathrm{Hz}, \mathrm{CH}_{2} \mathrm{CH}_{2} \mathrm{NH}_{3}{ }^{+}\right), 3.10\left(2 \mathrm{H}\right.$, br sextet, $\left.{ }^{3} J_{\mathrm{HH}}=7.2 \mathrm{~Hz}, \mathrm{CH}_{2} \mathrm{CH}_{2} \mathrm{NH}_{3}{ }^{+}\right), 3.60\left(3 \mathrm{H}, \mathrm{s}, \mathrm{CH}_{3}\right), 5.75$ $\left(1 \mathrm{H}, \mathrm{s}, 4-\mathrm{H}\right.$ of pyrazole), $8.23\left(3 \mathrm{H}, \mathrm{br} \mathrm{s}, \mathrm{NH}_{3}{ }^{+}\right)$, OH exchanged. ${ }^{13} \mathrm{C}$ NMR (125 MHz, DMSO- $\left.d_{6}\right)$, $\delta_{\mathrm{C}} 24.6,32.3,37.3,88.7,144.7,154.8 . \mathrm{MS}, \mathrm{m} / z=142\left(\mathrm{MH}^{+}\right)$, HRMS (ESI), $\mathrm{m} / z=142.0969$ $\left(\mathrm{MH}^{+}\right), \mathrm{C}_{6} \mathrm{H}_{12} \mathrm{~N}_{3} \mathrm{O}$ requires 142.0975. Anal. Calcd for $\mathrm{C}_{6} \mathrm{H}_{11} \mathrm{~N}_{3} \mathrm{O} \cdot 21 / 6 \mathrm{HCl}$ (220.17): C, 32.73; $\mathrm{H}$, 6.03; N, 19.09\%, Found: C, 32.67; H, 6.39; N, 18.90\%.

3-(2-Aminoethyl)-5-hydroxy-1-phenyl-1H-pyrazole dihydrochloride (6c). Prepared from 10c (1.515g, $5 \mathrm{mmol})$. White solid, yield $84 \%, 1.159 \mathrm{~g}, \mathrm{mp} 192-195^{\circ} \mathrm{C}$, IR $\left(v_{\max }, \mathrm{cm}^{-1}\right): 3381,1603$, 1547, 1497, 1464, 1366, 1310, 1145, 942, 812, 754, 691. ${ }^{1} \mathrm{H}$ NMR (300 MHz, DMSO- $\left.d_{6}\right), \delta_{\mathrm{H}}$ $2.92\left(2 \mathrm{H}, \mathrm{t},{ }^{3} \mathrm{~J}_{\mathrm{HH}}=7.5 \mathrm{~Hz}, \mathrm{CH}_{2} \mathrm{CH}_{2} \mathrm{NH}_{3}{ }^{+}\right), 3.13\left(2 \mathrm{H}\right.$, br sextet, $\left.{ }^{3} \mathrm{~J}_{\mathrm{HH}}=6.3 \mathrm{~Hz}, \mathrm{CH}_{2} \mathrm{CH}_{2} \mathrm{NH}_{3}{ }^{+}\right)$, $5.72\left(1 \mathrm{H}, \mathrm{s}, 4-\mathrm{H}\right.$ of pyrazole), $7.32\left(1 \mathrm{H}, \mathrm{br} \mathrm{t},{ }^{3} J_{\mathrm{HH}}=7.4 \mathrm{~Hz}, p-\mathrm{Ph}\right), 7.46\left(2 \mathrm{H}, \mathrm{br} \mathrm{t},{ }^{3} J_{\mathrm{HH}}=7.9 \mathrm{~Hz}\right.$, $m-\mathrm{Ph}), 7.71\left(2 \mathrm{H}\right.$, br d, $\left.{ }^{3} J_{\mathrm{HH}}=7.7 \mathrm{~Hz}, o-\mathrm{Ph}\right), 8.30\left(3 \mathrm{H}\right.$, br s, $\left.\mathrm{NH}_{3}{ }^{+}\right), \mathrm{OH}$ exchanged. ${ }^{13} \mathrm{C} \mathrm{NMR}$ (75.5 MHz, DMSO-d $d_{6}$ ), $\delta_{\mathrm{C}} 25.9,37.7,88.4,121.8,126.5,129.1,137.3,148.0,154.4 . \mathrm{MS}, \mathrm{m} / z=$ $204\left(\mathrm{MH}^{+}\right)$, HRMS (ESI), $m / z=204.1130\left(\mathrm{MH}^{+}\right), \mathrm{C}_{11} \mathrm{H}_{14} \mathrm{~N}_{3} \mathrm{O}$ requires 204.1131. Anal. Calcd for $\mathrm{C}_{11} \mathrm{H}_{13} \mathrm{~N}_{3} \mathrm{O} \cdot 2 \mathrm{HCl}$ (276.16): C, 47.84; H, 5.47; N, 15.22\%, Found: C, 47.22; H, 5.69; N, 14.95\%.

General procedures for acylation of amines 6b,c. Synthesis of 1-substituted 3-(2(acylamino)ethyl)-5-hydroxy-1H-pyrazoles (12b,c )and (13c)

Acetic anhydride $(0.1 \mathrm{~mL}, 1 \mathrm{mmol})$ or benzoyl chloride $(0.115 \mu \mathrm{L}, 1 \mathrm{mmol})$ was added to a stirred cold $\left(0{ }^{\circ} \mathrm{C}\right)$ solution of amine $6(1 \mathrm{mmol})$ and 4-methylmorpholine $(440 \mu \mathrm{L}, 4 \mathrm{mmol})$ in anhydrous methanol $(5 \mathrm{~mL})$ and the mixture was stirred at $0{ }^{\circ} \mathrm{C}$ for $1 \mathrm{~h}$ and then at r.t. for $12 \mathrm{~h}$. Volatile component were evaporated in vacuo and the residue was chromatographed over silica gel $(10 \% \mathrm{EtOH} / \mathrm{EtOAc})$. Fractions containing the product were combined and evaporated in vacuo to give $12 \mathrm{~b}, \mathrm{c}$ and $\mathbf{1 3 c}$.

$\boldsymbol{N}$-(2-(5-Hydroxy-1-methyl-1H-pyrazol-3-yl)ethyl)acetamide (12b). Prepared from 6b (214 $\mathrm{mg}, 1 \mathrm{mmol})$. White solid, yield $62 \%, 113 \mathrm{mg}, \mathrm{mp} 129-133{ }^{\circ} \mathrm{C}, \mathrm{IR}\left(v_{\max }, \mathrm{cm}^{-1}\right): 3255,3084$, 2953, 2884, 1633 (C=O), 1562, 1479, 1424, 1360, 1279, 1199, 1184, 1096, 1062, 1037, 894, 849, 768, 746, 716, 685, 674, 606. ${ }^{1} \mathrm{H}$ NMR (500 MHz, DMSO- $\left.d_{6}\right), \delta_{\mathrm{H}} 1.78(3 \mathrm{H}, \mathrm{s}, \mathrm{MeCO}), 2.46$ $\left(2 \mathrm{H}\right.$, br t, $\left.{ }^{3} J_{\mathrm{HH}}=7.5 \mathrm{~Hz}, \mathrm{CH}_{2} \mathrm{CH}_{2} \mathrm{NH}\right), 3.30\left(2 \mathrm{H}\right.$, br dt, $\left.{ }^{3} J_{\mathrm{HH}}=5.9,7.5 \mathrm{~Hz}, \mathrm{CH}_{2} \mathrm{CH}_{2} \mathrm{NH}\right), 3.41$ (3H, s, 1'-Me), 5.17 (1H, s, 4-H of pyrazole), $7.83\left(3 \mathrm{H}\right.$, br s, NH), $10.66\left(1 \mathrm{H}\right.$, br s OH). ${ }^{13} \mathrm{C} \mathrm{NMR}$ (126 MHz, DMSO- $d_{6}$ ), $\delta_{\mathrm{C}} 22.7,28.8,32.6,38.5,85.0,147.4,152.5,169.1 . \mathrm{MS}, \mathrm{m} / z=184$ $\left(\mathrm{MH}^{+}\right)$, HRMS (ESI), $m / z=184.1077\left(\mathrm{MH}^{+}\right), \mathrm{C}_{8} \mathrm{H}_{14} \mathrm{~N}_{3} \mathrm{O}_{2}$ requires 184.1081. Anal. Calcd for $\mathrm{C}_{8} \mathrm{H}_{13} \mathrm{~N}_{3} \mathrm{O}_{2}$ (183.21): C, 52.45; H, 7.15; N, 22.94\%, Found: C, 52.25; H, 7.38; N, 22.56\%. 
$\boldsymbol{N}$-(2-(5-Hydroxy-1-phenyl-1H-pyrazol-3-yl)ethyl)acetamide (12c). Prepared from 6c (276 $\mathrm{mg}, 5 \mathrm{mmol}$ ) and acetic anhydride. Yellowish solid, yield $87 \%, 213 \mathrm{mg}, \mathrm{mp} 150-155{ }^{\circ} \mathrm{C}$, IR $\left(v_{\max }, \mathrm{cm}^{-1}\right): 3312,1640(\mathrm{C}=\mathrm{O}), 1577,1558,1497,1398,1358,1308,1202,1150,787,751,687$. ${ }^{1} \mathrm{H}$ NMR $\left(500 \mathrm{MHz}, \mathrm{DMSO}-d_{6}\right), \delta_{\mathrm{H}} 1.80(3 \mathrm{H}, \mathrm{s}, \mathrm{Me}), 2.59\left(2 \mathrm{H}\right.$, br t, ${ }^{3} J_{\mathrm{HH}}=7.4 \mathrm{~Hz}$, $\left.\mathrm{CH}_{2} \mathrm{CH}_{2} \mathrm{NH}\right), 3.30\left(2 \mathrm{H}\right.$, br q, $\left.{ }^{3} J_{\mathrm{HH}}=7.3 \mathrm{~Hz}, \mathrm{CH}_{2} \mathrm{CH}_{2} \mathrm{NH}\right), 5.41(1 \mathrm{H}$, br s, 4-H of pyrazole $), 7.21$ $\left(1 \mathrm{H}\right.$, br t, $\left.{ }^{3} J_{\mathrm{HH}}=7.4 \mathrm{~Hz}, p-\mathrm{Ph}\right), 7.42\left(2 \mathrm{H}\right.$, br t, $\left.{ }^{3} J_{\mathrm{HH}}=8.0 \mathrm{~Hz}, m-\mathrm{Ph}\right), 7.72\left(2 \mathrm{H}, \mathrm{br} d,{ }^{3} J_{\mathrm{HH}}=7.8\right.$ $\mathrm{Hz}, o-\mathrm{Ph}), 7.90\left(3 \mathrm{H}\right.$, br s, NH), $11.46\left(1 \mathrm{H}\right.$, br s OH). ${ }^{13} \mathrm{C}$ NMR (126 MHz, DMSO- $\left.d_{6}\right), \delta_{\mathrm{C}} 22.7$, 28.9, 38.2, 86.9, 120.7, 125.1, 128.8, 138.9, 150.0, 153.0, 169.1. MS, $m / z=246\left(\mathrm{MH}^{+}\right)$, HRMS (ESI), $m / z=246.1231\left(\mathrm{MH}^{+}\right), \mathrm{C}_{13} \mathrm{H}_{16} \mathrm{~N}_{3} \mathrm{O}_{2}$ requires 246.1237. Anal. Calcd for $\mathrm{C}_{13} \mathrm{H}_{15} \mathrm{~N}_{3} \mathrm{O}_{2}$ (245.28): C, 63.66; H, 6.16; N, 17.13\%, Found: C, 63.47; H, 6.30; N, $16.91 \%$.

$\boldsymbol{N}$-(2-(5-Hydroxy-1-phenyl-1H-pyrazol-3-yl)ethyl)benzamide (13c). Prepared from 6c (276 $\mathrm{mg}, 1 \mathrm{mmol}$ ) and benzoyl chloride. Yellowish solid, yield 53\%, $163 \mathrm{mg}, \mathrm{mp} \mathrm{151-155}{ }^{\circ} \mathrm{C}$, IR $\left(v_{\max }, \mathrm{cm}^{-1}\right): 3280,1638(\mathrm{C}=\mathrm{O}), 1543,1495,1396,1312,1150,843,677 .{ }^{1} \mathrm{H}$ NMR $(500 \mathrm{MHz}$, DMSO-d $), \delta_{\mathrm{H}} 2.76\left(2 \mathrm{H}\right.$, br t, $\left.{ }^{3} J_{\mathrm{HH}}=7.4 \mathrm{~Hz}, \mathrm{CH}_{2} \mathrm{CH}_{2} \mathrm{NH}\right), 3.54\left(2 \mathrm{H}\right.$, br q, ${ }^{3} J_{\mathrm{HH}}=7.3 \mathrm{~Hz}$, $\left.\mathrm{CH}_{2} \mathrm{CH}_{2} \mathrm{NH}\right), 5.47\left(1 \mathrm{H}\right.$, br s, 4-H of pyrazole), $7.22\left(1 \mathrm{H}\right.$, br t, $\left.{ }^{3} J_{\mathrm{HH}}=7.4 \mathrm{~Hz}, p-\mathrm{Ph}\right), 7.40-7.49$ $(4 \mathrm{H}, \mathrm{m}, 2 \times m-\mathrm{Ph}), 7.50-7.55(1 \mathrm{H}, \mathrm{m}, p-\mathrm{Ph}), 7.73$ and $7.86\left(4 \mathrm{H}, 2 \mathrm{br} \mathrm{d}, 1: 1,{ }^{3} J_{\mathrm{HH}}=7.5 \mathrm{~Hz}, 2 \times o-\right.$ $\mathrm{Ph}), 8.60\left(1 \mathrm{H}\right.$, br s, NH), $11.55\left(1 \mathrm{H}\right.$, br s, OH). ${ }^{1} \mathrm{H}$ NMR $\left(500 \mathrm{MHz}, \mathrm{CDCl}_{3}\right), \delta_{\mathrm{H}} 2.81(2 \mathrm{H}$, br t, $\left.{ }^{3} J_{\mathrm{HH}}=6.2 \mathrm{~Hz}, \mathrm{CH}_{2} \mathrm{CH}_{2} \mathrm{NH}\right), 3.51\left(2 \mathrm{H}\right.$, br s, $4-\mathrm{CH}_{2}$ of pyrazole), $3.88\left(2 \mathrm{H}, \mathrm{br} \mathrm{q},{ }^{3} J_{\mathrm{HH}}=6.2 \mathrm{~Hz}\right.$, $\left.\mathrm{CH}_{2} \mathrm{CH}_{2} \mathrm{NH}\right), 6.75\left(1 \mathrm{H}\right.$, br s, NH), $7.19\left(1 \mathrm{H}\right.$, br t, $\left.{ }^{3} J_{\mathrm{HH}}=7.4 \mathrm{~Hz}, p-\mathrm{Ph}\right), 7.36-7.44(4 \mathrm{H}, \mathrm{m}, 2 \times m-$ $\mathrm{Ph}), 7.45-7.52(1 \mathrm{H}, \mathrm{m}, p-\mathrm{Ph}), 7.72-7.77$ and 7.80-7.86 (4H, 2m, 1:1, 2×o-Ph). ${ }^{13} \mathrm{C}$ NMR (126 MHz, DMSO- $\left.d_{6}\right), \delta_{\mathrm{C}} 28.8,39.0,87.0,118.0,120.7,125.2,127.2,128.3,128.8,131.1,134.6$, 150.0, 153.0, 166.1. MS, $m / z=308\left(\mathrm{MH}^{+}\right)$, HRMS (ESI), $m / z=308.1385\left(\mathrm{MH}^{+}\right), \mathrm{C}_{18} \mathrm{H}_{18} \mathrm{~N}_{3} \mathrm{O}_{2}$ requires 308.1394. Anal. Calcd for $\mathrm{C}_{18} \mathrm{H}_{17} \mathrm{~N}_{3} \mathrm{O}_{2}$ (307.35): C, 70.34; H, 5.58; N, 13.67\%, Found: C, 70.18; H, 5.60; N, $13.35 \%$.

3-(2-(Benzoylamino)ethyl)-1-phenyl-1H-pyrazol-5-yl benzoate (14). Benzoyl chloride (0.230 $\mathrm{mL}, 2 \mathrm{mmol})$ was added to a stirred suspension of amine $\mathbf{6 c}(276 \mathrm{mg}, 1 \mathrm{mmol})$ in a mixture of anhydrous dichloromethane $(10 \mathrm{~mL})$ and 4-methylmorpholine $(0.66 \mathrm{~mL}, 6 \mathrm{mmol})$ and the mixture was stirred at r.t. for $12 \mathrm{~h}$. Volatile component were evaporated in vacuo and the residue was chromatographed over silica gel (50\% EtOAc/hexanes). Fractions containing the products were combined and evaporated in vacuo to give 14. Yellow solid, yield 79\%, $326 \mathrm{mg}$, mp 109$112{ }^{\circ} \mathrm{C}$, IR $\left(v_{\max }, \mathrm{cm}^{-1}\right): 3289,1753(\mathrm{C}=\mathrm{O}), 1632(\mathrm{C}=\mathrm{O}), 1445,1315,1247,1076,760,697 .{ }^{1} \mathrm{H}$ NMR $\left(300 \mathrm{MHz}, \mathrm{CDCl}_{3}\right), \delta_{\mathrm{H}} 3.01\left(2 \mathrm{H}, \mathrm{t},{ }^{3} \mathrm{~J}_{\mathrm{HH}}=6.3 \mathrm{~Hz}, \mathrm{CH}_{2} \mathrm{CH}_{2} \mathrm{NH}\right), 3.86\left(2 \mathrm{H}, \mathrm{q},{ }^{3} J_{\mathrm{HH}}=6.0\right.$ $\left.\mathrm{Hz}, \mathrm{CH}_{2} \mathrm{CH}_{2} \mathrm{NH}\right), 6.36(1 \mathrm{H}, \mathrm{s}, 4-\mathrm{H}$ of pyrazole), $7.16(3 \mathrm{H}$, br s, $\mathrm{NH}), 7.31-7.53(8 \mathrm{H}, \mathrm{m}, 8 \mathrm{H}$ of $\mathrm{Ph}), 7.59-7.68(3 \mathrm{H}, \mathrm{m}, 3 \mathrm{H}$ of $\mathrm{Ph}), 7.78-7.83$ (2H, m, 2H of $\mathrm{Ph}), 8.05-8.11$ (2H, m, 2H of Ph). ${ }^{13} \mathrm{C}$ NMR (126 MHz, DMSO- $\left.d_{6}\right), \delta_{\mathrm{C}} 28.7,38.9,95.8,122.7,127.17,127.23,127.3,128.3$, $129.25,129.30,130.1,131.1,134.6,134.8,137.6,144.0,150.3,161.8,166.2 . \mathrm{MS}, \mathrm{m} / \mathrm{z}=412$ $\left(\mathrm{MH}^{+}\right)$, HRMS (ESI), $m / z=412.1649\left(\mathrm{MH}^{+}\right), \mathrm{C}_{25} \mathrm{H}_{22} \mathrm{~N}_{3} \mathrm{O}_{3}$ requires 412.1656. Anal. Calcd for $\mathrm{C}_{25} \mathrm{H}_{21} \mathrm{~N}_{3} \mathrm{O}_{3}$ (411.45): C, 63.66; H, 6.16; N, 17.13\%, Found: C, 63.47; H, 6.30; N, 16.91\%. 


\section{General procedure for the synthesis of $N, N$-dialkyl- $\beta$-alanines $(19 \mathrm{a}, \mathrm{b})$}

First, the esters $\mathbf{1 8 a}$ and $\mathbf{1 8 b}$ were prepared following slightly modified literature procedure. ${ }^{10 \mathrm{a}}$ Amine $17(141 \mathrm{mmol})$ was added to a cooled $\left(0^{\circ} \mathrm{C}\right)$ solution of methyl acrylate $16(12.7 \mathrm{~mL}, 141$ $\mathrm{mmol})$ in dichloromethane $(150 \mathrm{~mL})$ and the reaction mixture was stirred at room temperature for 2-24 h. The solvent was evaporated in vacuo to yield the $\beta$-amino ester $\mathbf{1 8}$ as a yellowish oil, which was characterized by ${ }^{1} \mathrm{H}$ NMR.

Then, $4.4 \mathrm{M}$ aq. $\mathrm{NaOH}(100 \mathrm{~mL}, 440 \mathrm{mmol})$ was added to the ester 18 (141 $\mathrm{mmol})$ and the mixture was vigorously stirred at r.t. for $2-5 \mathrm{~h}$. Reaction mixture was cooled to $0{ }^{\circ} \mathrm{C}$ and neutralized with $6 \mathrm{M}$ aq. $\mathrm{HCl}(73.3 \mathrm{~mL})$, stirred at r.t. for $15 \mathrm{~min}$, and washed with EtOAc (50 $\mathrm{mL})$ to remove non-polar impurities. The aqueous phase was purified by ion-exchange chromatography over Dowex ${ }^{\circledR} 50 \mathrm{~W}$ cation exchange resin. The product was eluted from the resin with $4 \%$ aq. ammonia $(1 \mathrm{~L})$, volatile components were evaporated in vacuo, and the residue was re-suspended five times in EtOH $(100 \mathrm{~mL})$ to remove $\mathrm{H}_{2} \mathrm{O}$ and five times in $\mathrm{CH}_{2} \mathrm{Cl}_{2}(100$ $\mathrm{mL}$ ) to remove $\mathrm{EtOH}$. The residue was dried in vacuo $\left(0.01 \mathrm{Torr}, 40{ }^{\circ} \mathrm{C}\right)$ to give the $\beta$-amino acids 19a,b, which were characterized by NMR.

Methyl 3-(benzyl(methyl)amino)propanoate (18a). ${ }^{10 a}$ Prepared from 16 (12.7 mL, $\left.141 \mathrm{mmol}\right)$ and benzyl(methyl)amine $17 \mathbf{a}(18.9 \mathrm{~mL}, 141 \mathrm{mmol})$, stirring for $24 \mathrm{~h}$. Yellowish oil, yield: 100\%, 29.02 g. ${ }^{1} \mathrm{H}$ NMR (300 MHz, $\left.\mathrm{CDCl}_{3}\right): \delta 2.20(3 \mathrm{H}, \mathrm{s}, \mathrm{NMe}), 2.51(2 \mathrm{H}, \mathrm{t}, J=7.1 \mathrm{~Hz}, 2-$ $\left.\mathrm{CH}_{2}\right), 2.74\left(2 \mathrm{H}, \mathrm{t}, J=7.1 \mathrm{~Hz}, 2-\mathrm{CH}_{2}\right), 3.50\left(2 \mathrm{H}, \mathrm{s}, \mathrm{CH}_{2} \mathrm{Ph}\right), 3.66(3 \mathrm{H}, \mathrm{s}, \mathrm{OMe}), 7.20-7.35(5 \mathrm{H}$, $\mathrm{m}, \mathrm{Ph})$.

Methyl 3-(pyrrolidin-1-yl)propanoate (18b). ${ }^{10 \mathrm{~b}, \mathrm{c}}$ Prepared from $16(12.7 \mathrm{~mL}, 141 \mathrm{mmol})$ and pyrrolidine $17 \mathbf{a}(11.8 \mathrm{~mL}, 141 \mathrm{mmol})$, stirring for $2 \mathrm{~h}$. Yellowish oil, yield: $100 \%, 22.01 \mathrm{~g} .{ }^{1} \mathrm{H}$ NMR $\left(300 \mathrm{MHz}, \mathrm{CDCl}_{3}\right): \delta 1.74-1.82\left(4 \mathrm{H}, \mathrm{m}, 3^{\prime}-\mathrm{CH}_{2}\right.$ and $\left.4^{\prime}-\mathrm{CH}_{2}\right), 2.47-2.58\left(6 \mathrm{H}, \mathrm{m}, 2^{\prime}-\mathrm{CH}_{2}\right.$, 5'- $\mathrm{CH}_{2}$, and 2- $\left.\mathrm{CH}_{2}\right), 2.74-2.81\left(2 \mathrm{H}, \mathrm{m}, 3-\mathrm{CH}_{2}\right), 3.68(3 \mathrm{H}, \mathrm{s}, \mathrm{OMe})$.

3-(Benzyl(methyl)amino)propanoic acid (19a). ${ }^{11 a}$ Prepared from methyl 3(benzyl(methyl)amino)propanoate 18a $(29.02 \mathrm{~g}, 140 \mathrm{mmol})$, stirring for $5 \mathrm{~h}$. Brownish semisolid, yield: 77\%, 22.12 g. ${ }^{1} \mathrm{H}$ NMR $\left(300 \mathrm{MHz}, \mathrm{CDCl}_{3}\right): \delta 2.17(3 \mathrm{H}, \mathrm{s}, \mathrm{NMe}), 2.45\left(2 \mathrm{H}, \mathrm{t},{ }^{3} J_{\mathrm{HH}}=\right.$ $\left.7.1 \mathrm{~Hz}, 2-\mathrm{CH}_{2}\right), 2.80\left(2 \mathrm{H}, \mathrm{t},{ }^{3} J_{\mathrm{HH}}=7.1 \mathrm{~Hz}, 3-\mathrm{CH}_{2}\right), 3.60\left(2 \mathrm{H}, \mathrm{s}, \mathrm{CH}_{2} \mathrm{Ph}\right), 7.15-7.31(5 \mathrm{H}, \mathrm{m}, \mathrm{Ph})$, $10.69(1 \mathrm{H}$, br s, $\mathrm{COOH}) .{ }^{13} \mathrm{C} \mathrm{NMR}\left(75 \mathrm{MHz}, \mathrm{CDCl}_{3}\right): \delta 33.7,40.75,53.7,61.1,127.8,128.59$, 129.9, 136.1, 178.0.

3-(Pyrrolidin-1-yl)propanoic acid (19b). ${ }^{11 \mathrm{~b}}$ Prepared from methyl 3-(pyrrolidin-1yl)propanoate $\mathbf{1 8 b}(22.01 \mathrm{~g}, 140 \mathrm{mmol})$, stirring for $2 \mathrm{~h}$. Brownish semi-solid, yield: 80\%, 16.19 g. ${ }^{1} \mathrm{H}$ NMR (300 MHz, $\left.\mathrm{D}_{2} \mathrm{O}\right): \delta$ 1.90-2.06 $\left(4 \mathrm{H}, \mathrm{m}, 3^{\prime}-\mathrm{CH}_{2}\right.$ and $\left.4{ }^{\prime}-\mathrm{CH}_{2}\right), 2.54\left(2 \mathrm{H}, \mathrm{t},{ }^{3} J_{\mathrm{HH}}=7.3\right.$ $\left.\mathrm{Hz}, 2-\mathrm{CH}_{2}\right), 3.08-3.24\left(6 \mathrm{H}, \mathrm{m}, 3-\mathrm{CH}_{2}, 2^{\prime}-\mathrm{CH}_{2}\right.$, and $\left.5^{\prime}-\mathrm{CH}_{2}\right), \mathrm{COOH}$ exchanged. ${ }^{13} \mathrm{C}$ NMR $(75$ $\left.\mathrm{MHz}, \mathrm{D}_{2} \mathrm{O}\right): \delta 23.1,34.4,52.3,53.9,178.5$.

Synthesis of methyl 2-(1-(4-methoxyphenyl)-5-oxo-4,5-dihydro-1H-pyrazol-3-yl)acetate (22b). A mixture of 21 (0.803 mL, $5.28 \mathrm{mmol})$, (4-methoxyphenyl)hydrazine hydrochloride 9h (922 $\mathrm{mg}, 5.28 \mathrm{mmol})$, toluene $(15 \mathrm{~mL})$, and $\mathrm{Et}_{3} \mathrm{~N}(0.736 \mathrm{~mL}, 5.28 \mathrm{mmol})$ was stirred at r.t. for $4 \mathrm{~h}$ and then at $80{ }^{\circ} \mathrm{C}$ for additional $2 \mathrm{~h}$. The reaction mixture was purified directly by $\mathrm{CC}$ 
(hexanes/EtOAc, 1:1) without the prior removal of volatile components (mainly toluene). Fractions containing the product were combined and evaporated in vacuo. The solid residue was re-crystallized from a mixture of EtOAc/ $n$-heptane to give 22b. White solid, yield: 43\%, $600 \mathrm{mg}$, mp 141-142 ${ }^{\circ} \mathrm{C}$, IR $\left(v_{\max }, \mathrm{cm}^{-1}\right): 3448,1744(\mathrm{C}=\mathrm{O}), 1637(\mathrm{C}=\mathrm{O}), 1542,1516,1465,1406,1314$, $1252,1173,1150,1111,1032,1005,840,756,630 .{ }^{1} \mathrm{H} \mathrm{NMR}\left(300 \mathrm{MHz}, \mathrm{CDCl}_{3}\right): \delta_{\mathrm{H}} 3.58$ and $3.60\left(4 \mathrm{H}, 2 \mathrm{~s}, 1: 1,2-\mathrm{CH}_{2}, 4-\mathrm{CH}_{2}\right.$ of pyrazole), 3.77 and $3.81(6 \mathrm{H}, 2 \mathrm{~s}, 1: 1,2 \times \mathrm{OMe}), 6.88-6.96$ $\left(2 \mathrm{H}, \mathrm{m}, m-\mathrm{C}_{6} \mathrm{H}_{4}\right), 7.67-7.75\left(2 \mathrm{H}, \mathrm{m}, o-\mathrm{C}_{6} \mathrm{H}_{4}\right) .{ }^{1} \mathrm{H}$ NMR $\left(500 \mathrm{MHz}, \mathrm{DMSO}-d_{6}\right): \delta_{\mathrm{H}} 3.54(2 \mathrm{H}, \mathrm{s}$, $\mathrm{CH}_{2}$ ), 3.63 and $3.77(6 \mathrm{H}, 2 \mathrm{~s}, 1: 1,2 \times \mathrm{OMe}), 5.47(1 \mathrm{H}, \mathrm{s}, 4-\mathrm{H}$ of pyrazole), 6.99 and 7.57 (4H, 2td, $\left.1: 1,{ }^{4} J_{\mathrm{HH}}=2.8 \mathrm{~Hz},{ }^{3} J_{\mathrm{HH}}=9.1 \mathrm{~Hz}, \mathrm{C}_{6} \mathrm{H}_{4}\right), 11.44(1 \mathrm{H}, \mathrm{s}, \mathrm{OH}) .{ }^{13} \mathrm{C}$ NMR $\left(126 \mathrm{MHz}, \mathrm{DMSO}-d_{6}\right): \delta_{\mathrm{C}}$ 34.6, 51.7, 55.3, 87.4, 114.0, 122.7, 132.0, 144.6, 152.6, 157.0, 170.7. MS, $m / z=263\left(\mathrm{MH}^{+}\right)$, HRMS (ESI), $m / z=263.1035\left(\mathrm{MH}^{+}\right), \mathrm{C}_{13} \mathrm{H}_{15} \mathrm{~N}_{2} \mathrm{O}_{4}$ requires 263.1032. Anal. Calcd for $\mathrm{C}_{13} \mathrm{H}_{14} \mathrm{~N}_{2} \mathrm{O}_{4}$ (262.26): C, 59.54; H, 5.38; N, 10.68\%, Found: C, 59.54; H, 5.14; N, $10.61 \%$.

\section{General procedure for the preparation of carboxamides (24a) and (25b,c)}

A suspension of the ester $22(10 \mathrm{mmol})$ in $1.5 \mathrm{M}$ aq. $\mathrm{NaOH}(40 \mathrm{~mL})$ was vigorously stirred at r.t. for $24 \mathrm{~h}$. Reaction mixture was neutralized with $3 \mathrm{M}$ aq. $\mathrm{HCl}(20 \mathrm{~mL})$ and stirred for additional $15 \mathrm{~min}$ at r.t. The product was extracted with EtOAc $(3 \times 100 \mathrm{~mL})$, the combined organic phase was dried over anh. $\mathrm{Na}_{2} \mathrm{SO}_{4}$, filtered, and the volatile components were evaporated in vacuo to give 23a,b as sticky resins, which were characterised by ${ }^{1} \mathrm{H}$ NMR and HRMS and then used for further amidation without purification. Under argon, the crude acid 23 (10 mmol) was dissolved in anh. THF (40 mL), 1,1'-carbonyldiimidazole (2.432 g, $15 \mathrm{mmol})$ was added, and the reaction mixture was stirred at r. t. for $2 \mathrm{~h}$. Then, the corresponding amine 17 (40 mmol) was added and stirring at r.t. was continued for $3 \mathrm{~h}$. Volatile components were evaporated in vacuo, the residue was dissolved in EtOAc $(200 \mathrm{~mL})$ and the resulting solution was washed with $1 \mathrm{M}$ aq. $\mathrm{NaHSO}_{4}$ $(100 \mathrm{~mL})$. The organic phase was dried over anh. $\mathrm{Na}_{2} \mathrm{SO}_{4}$, filtered, and volatile components were evaporated in vacuo. The residue was purified by $\mathrm{CC}$ (EtOAc/hexanes). Fractions containing the product were combined and volatile components were evaporated in vacuo to give $\mathbf{2 4}$ or $\mathbf{2 5}$ as a viscous oil.

2-(5-Hydroxy-1-phenyl-1H-pyrazol-3-yl)acetic acid (23a). Prepared from 21a (2.46 g, 10 mmol). Yellow-orange resin, yield 94\%, 2.064 g. ${ }^{1} \mathrm{H}$ NMR $\left(300 \mathrm{MHz}, \mathrm{DMSO}-d_{6}\right), \delta_{\mathrm{H}} 3.45(2 \mathrm{H}$, s, $\left.\mathrm{CH}_{2}\right), 5.49$ (1H, s, 4-H of pyrazole), 7.18-7.28 (1H, m, p-Ph), 7.38-7.49 (2H, m, m-Ph), 7.68$7.74(2 \mathrm{H}, \mathrm{m}, o-\mathrm{Ph}), 11.68(1 \mathrm{H}$, br s, OH$), 12.25\left(1 \mathrm{H}\right.$, br s, OH). MS, $m / z=219\left(\mathrm{MH}^{+}\right), \mathrm{HRMS}$ $(\mathrm{ESI}), m / z=219.0766\left(\mathrm{MH}^{+}\right), \mathrm{C}_{11} \mathrm{H}_{11} \mathrm{~N}_{2} \mathrm{O}_{3}$ requires 219.0770 .

2-(5-Hydroxy-1-(4-methoxyphenyl)-1H-pyrazol-3-yl)acetic acid (23b). Prepared from 21b (2.625 g, $10 \mathrm{mmol}$ ). Yellow-brown resin, yield 79\%, 1.979 g. ${ }^{1} \mathrm{H}$ NMR (300 MHz, DMSO-d6), $\delta_{\mathrm{H}} 3.42\left(2 \mathrm{H}, \mathrm{s}, \mathrm{CH}_{2}\right), 3.77(3 \mathrm{H}, \mathrm{s}, \mathrm{OMe}), 5.45(1 \mathrm{H}, \mathrm{s}, 4-\mathrm{H}$ of pyrazole), 6.95-7.03 and 7.52-7.61 $\left(4 \mathrm{H}, 2 \mathrm{~m}, 1: 1, \mathrm{C}_{6} \mathrm{H}_{4}\right), 11.34\left(1 \mathrm{H}\right.$, br s, OH), $12.29(1 \mathrm{H}$, br s, COOH$) . \mathrm{MS}, m / z=249\left(\mathrm{MH}^{+}\right)$, HRMS (ESI), $m / z=249.0867\left(\mathrm{MH}^{+}\right), \mathrm{C}_{12} \mathrm{H}_{13} \mathrm{~N}_{2} \mathrm{O}_{4}$ requires 219.0875 . 
$N$-Benzyl-2-(5-hydroxy-1-phenyl-1H-pyrazol-3-yl)- $N$-methylacetamide (24a). Prepared from 23a (2.181 g, $10 \mathrm{mmol}$ ) and benzyl(methyl)amine 17a (4.844 g, $40 \mathrm{mmol}), \mathrm{CC}$ (EtOAc/hexanes, 2:1). Yellowish oil, yield 48\%, $1.561 \mathrm{~g}$, IR $\left(v_{\max }, \mathrm{cm}^{-1}\right): 3490,1639(\mathrm{C}=\mathrm{O}), 1559,1496,1453$, 1401, 1112, 1020, 804, 759, 731, 692. ${ }^{1} \mathrm{H}$ NMR (300 MHz, $\left.\mathrm{CDCl}_{3}\right), \delta_{\mathrm{H}} 3.01$ (3H, s, Me), 3.63$3.72\left(4 \mathrm{H}, \mathrm{m}, 2-\mathrm{CH}_{2}\right.$ and $4-\mathrm{CH}_{2}$ of pyrazole $), 4.61$ and $4.63\left(2 \mathrm{H}, 2 \mathrm{br} \mathrm{s}, 5: 3, \mathrm{CH}_{2} \mathrm{Ph}\right), 7.14-7.22$ $(2 \mathrm{H}, \mathrm{m}, 2 \mathrm{H}$ of $\mathrm{Ph}), 7.24-7.42(6 \mathrm{H}, \mathrm{m}, 6 \mathrm{H}$ of $\mathrm{Ph}), 7.77-7.86(2 \mathrm{H}, \mathrm{m}, 2 \mathrm{H}$ of $\mathrm{Ph}) .{ }^{13} \mathrm{C} \mathrm{NMR}(300$ $\left.\mathrm{MHz}, \mathrm{CDCl}_{3}\right), \quad \delta_{\mathrm{C}} 33.8 / 34.0,35.9 / 36.2,42.1 / 42.2,50.8 / 50.9,118.58 / 118.60,121.3 / 121.4$, 127.5/127.6, 127.3/127.4, 128.3/128.4, 128.5/128.6, 136.3/136.4, 137.7/137.8, 154.6/154.7, 167.7/167.9, 170.7/170.9. ${ }^{1} \mathrm{H}$ NMR (500 MHz, DMSO- $\left.d_{6}\right), \delta_{\mathrm{H}} 2.79$ and $2.98(3 \mathrm{H}, 2 \mathrm{~s}, 1: 2, \mathrm{Me})$, 3.62 and $3.67\left(2 \mathrm{H}, 2 \mathrm{~s}, 1: 2,2-\mathrm{CH}_{2}\right), 4.54$ and $4.68\left(2 \mathrm{H}, 2 \mathrm{~s}, 2: 1, \mathrm{CH}_{2} \mathrm{Ph}\right), 5.47$ and $5.48(1 \mathrm{H}, 2 \mathrm{~s}$, $\sim 1: 2$, 4-H of pyrazole), 7.16-7.36 (8H, m, 8H of Ph), 7.38-7.46 (2H, m, 2H of Ph), 7.64-7.67 and 7.72-7.75 $(1 \mathrm{H}, 2 \mathrm{~m}, 1: 2,1 \mathrm{H}$ of $\mathrm{Ph}), 11.63\left(1 \mathrm{H}\right.$, br s, OH). ${ }^{13} \mathrm{C}$ NMR (126 MHz, DMSO- $\left.d_{6}\right)$, $\delta_{\mathrm{C}} 33.4 / 35.3,34.6 / 34.8,50.0 / 52.8,87.7,120.6 / 120.9,125.3 / 125.4,126.8 / 127.4,127.0 / 127.3$, $128.4 / 128.6,128.8 / 128.9,137.4 / 137.8,138.8 / 138.9,146.7,153.2,169.4 / 169.5 . \mathrm{MS}, \mathrm{m} / \mathrm{z}=322$ $\left(\mathrm{MH}^{+}\right)$, HRMS (ESI), $m / z=322.1549\left(\mathrm{MH}^{+}\right), \mathrm{C}_{19} \mathrm{H}_{20} \mathrm{~N}_{3} \mathrm{O}_{2}$ requires 322.1556.

\section{1-(4-Methoxyphenyl)-3-(2-oxo-2-(pyrrolidin-1-yl)ethyl)-1H-pyrazol-5(4H)-one}

(25b).

Prepared from 23b $(2.491 \mathrm{~g}, 10 \mathrm{mmol})$ and pyrrolidine $\mathbf{1 7 b}(2.840 \mathrm{~g}, 40 \mathrm{mmol}), \mathrm{CC}$ (first EtOAc to elute less polar impurities, then $\mathrm{MeOH} / \mathrm{EtOAc}, 1: 9$, to elute the product). Yellowish oil, yield 79\%, $2.388 \mathrm{~g}$, IR $\left(v_{\max }, \mathrm{cm}^{-1}\right): 3438,2928,2877,1703(\mathrm{C}=\mathrm{O}), 1621,1557,1511,1454,1340$, 1298, 1249, 1172, 1024, 913, 833, 730. ${ }^{1} \mathrm{H}$ NMR (500 MHz, $\left.\mathrm{CDCl}_{3}\right), \delta_{\mathrm{H}} 1.89$ and 2.00 (4H, 2 quintets, $1: 1,{ }^{3} J_{\mathrm{HH}}=6.8 \mathrm{~Hz}, 3$ "- $\left.\mathrm{CH}_{2}, 4 "-\mathrm{CH}_{2}\right), 3.49$ and $3.51\left(4 \mathrm{H}, 2 \mathrm{t}, 1: 1,{ }^{3} \mathrm{~J}_{\mathrm{HH}}=6.9 \mathrm{~Hz}, 2 "-\mathrm{CH}_{2}\right.$, 5"- $\left.\mathrm{CH}_{2}\right), 3.53$ and $3.68\left(4 \mathrm{H}, 2 \mathrm{~s}, 1: 1,2{ }^{\prime}-\mathrm{CH}_{2}, 4-\mathrm{CH}_{2}\right.$ of pyrazole), 3.81 (3H, s, OMe), 6.91 and $7.70\left(4 \mathrm{H}, 2 \mathrm{br} \mathrm{d}, 1: 1,{ }^{3} J_{\mathrm{HH}}=9.1 \mathrm{~Hz}, \mathrm{C}_{6} \mathrm{H}_{4}\right) .{ }^{1} \mathrm{H} \mathrm{NMR}\left(300 \mathrm{MHz}, \mathrm{DMSO}-d_{6}\right), \delta_{\mathrm{H}} 1.70-1.93(4 \mathrm{H}$, m, 3"- $\mathrm{CH}_{2}, 4$ "- $\left.\mathrm{CH}_{2}\right), 3.28$ and $3.50\left(4 \mathrm{H}, 2 \mathrm{t},{ }^{3} \mathrm{~J}_{\mathrm{HH}}=6.9 \mathrm{~Hz}, 2^{\prime \prime}-\mathrm{CH}_{2}, 5\right.$ "- $\left.\mathrm{CH}_{2}\right), 3.46\left(2 \mathrm{H}, \mathrm{s}, 1\right.$ '- $\left.-\mathrm{CH}_{2}\right)$, $3.77(3 \mathrm{H}, \mathrm{s}, \mathrm{OMe}), 5.40\left(1 \mathrm{H}, \mathrm{s}, 4-\mathrm{H}\right.$ of pyrazole), 6.95-7.03 and 7.52-7.60 (4H, 2m, 1:1, $\left.\mathrm{C}_{6} \mathrm{H}_{4}\right)$, $11.31\left(1 \mathrm{H}\right.$, br s, OH). ${ }^{13} \mathrm{C} \mathrm{NMR}\left(126 \mathrm{MHz}, \mathrm{CDCl}_{3}\right), \delta_{\mathrm{C}} 24.5,26.2,37.8,42.5,46.2,47.1,55.6$, $114.1,121.1,131.4,154.5,157.2,166.0,170.9 . \mathrm{MS}, \mathrm{m} / z=302\left(\mathrm{MH}^{+}\right)$, HRMS $(\mathrm{ESI}), \mathrm{m} / z=$ $302.1510\left(\mathrm{MH}^{+}\right), \mathrm{C}_{16} \mathrm{H}_{20} \mathrm{~N}_{3} \mathrm{O}_{3}$ requires 302.1505.

$\mathrm{N}, \boldsymbol{N}$-dibenzyl-2-(1-phenyl-5-oxo-4,5-dihydro-1H-pyrazol-3-yl)acetamide $\quad \mathbf{( 2 5 c )}$. Prepared from 23a (2.181 g, $10 \mathrm{mmol})$ and dibenzylamine 17c $(7.88 \mathrm{~g}, 40 \mathrm{mmol}), \mathrm{CC}$ (EtOAc/hexanes, 1:1). Yellowish semi-solid, yield 42\%, $1.675 \mathrm{~g}$, IR $\left(v_{\max }, \mathrm{cm}^{-1}\right): 3480,1715(\mathrm{C}=\mathrm{O}), 1633(\mathrm{C}=\mathrm{O})$, 1558, 1496, 1452, 1360, 1208, 1168, 1078, 954, 751, 694. ${ }^{1} \mathrm{H}$ NMR (300 MHz, $\left.\mathrm{CDCl}_{3}\right), \delta_{\mathrm{H}} 3.65$ $\left(4 \mathrm{H}, \mathrm{s}, 2-\mathrm{CH}_{2}, 4-\mathrm{CH}_{2}\right.$ of pyrazole), 4.52 and $4.64\left(4 \mathrm{H}, 2 \mathrm{~s}, 1: 1,2 \times \mathrm{CH}_{2} \mathrm{Ph}\right), 7.12-7.42(13 \mathrm{H}, \mathrm{m}$, $13 \mathrm{H}$ of $\mathrm{Ph}), 7.77-7.83$ (2H, m, 2H of Ph). ${ }^{1} \mathrm{H}$ NMR (500 MHz, DMSO-d $)$, $\delta_{\mathrm{H}} 3.68$ (2H, 2s, 1:2, 2- $\left.\mathrm{CH}_{2}\right), 4.51$ and $4.60\left(4 \mathrm{H}, 2 \mathrm{~s}, 1: 1, \mathrm{CH}_{2} \mathrm{Ph}\right), 5.51(1 \mathrm{H}, \mathrm{s}, 4-\mathrm{H}$ of pyrazole $), 7.19\left(3 \mathrm{H}, \mathrm{d},{ }^{3} J_{\mathrm{HH}}=\right.$ $7.1 \mathrm{~Hz}, 3 \mathrm{H}$ of $\mathrm{Ph}), 7.22-7.30(6 \mathrm{H}, \mathrm{m}, 6 \mathrm{H}$ of $\mathrm{Ph}), 7.35\left(2 \mathrm{H}, \mathrm{t},{ }^{3} \mathrm{~J}_{\mathrm{HH}}=7.4 \mathrm{~Hz}, 2 \mathrm{H}\right.$ of $\left.\mathrm{Ph}\right), 7.43(2 \mathrm{H}$, $\mathrm{dd},{ }^{3} J_{\mathrm{HH}}=7.3,8.6 \mathrm{~Hz}, 2 \mathrm{H}$ of $\left.\mathrm{Ph}\right), 7.70\left(2 \mathrm{H}, \mathrm{dd},{ }^{3} J_{\mathrm{HH}}=1.4,8.3 \mathrm{~Hz}, 2 \mathrm{H}\right.$ of $\left.\mathrm{Ph}\right), 11.67(1 \mathrm{H}$, br s, $\mathrm{OH}) .{ }^{13} \mathrm{C}$ NMR $\left(126 \mathrm{MHz}, \mathrm{DMSO}-d_{6}\right), \delta_{\mathrm{C}} 34.8,47.7,50.4,87.7,120.8,125.4,126.7,127.0$, $127.4,127.5,128.4,128.7,128.8,137.2,137.6,138.8,146.6,153.2,169.9 . \mathrm{MS}, \mathrm{m} / z=398$ $\left(\mathrm{MH}^{+}\right), \mathrm{HRMS}(\mathrm{ESI}), \mathrm{m} / z=398.1859\left(\mathrm{MH}^{+}\right), \mathrm{C}_{25} \mathrm{H}_{24} \mathrm{~N}_{3} \mathrm{O}_{2}$ requires 398.1869. 
General procedure for the reduction of amides $(24 a)$ and $(25 b, c)$ to amines $(15 a, b)$ and (26c)

Under argon, $\mathrm{LiAlH}_{4}(1 \mathrm{M}$ in THF, $10 \mathrm{~mL}, 10 \mathrm{mmol})$ was added to a cooled $\left(0{ }^{\circ} \mathrm{C}\right)$ solution of amide 24 or $25(5 \mathrm{mmol})$ in anhydrous THF $(10 \mathrm{~mL})$ and the resulting reaction mixture was stirred at $0{ }^{\circ} \mathrm{C}$ for $20 \mathrm{~min}$. and then at $60^{\circ} \mathrm{C}$ for $5 \mathrm{~h}$. The reaction mixture was cooled to $0{ }^{\circ} \mathrm{C}$ and quenched subsequently with aq. sat. $\mathrm{NaHCO}_{3}(5 \mathrm{~mL})$ and $\mathrm{MeOH}(10 \mathrm{~mL})$. The resulting mixture was stirred at room temperature for $10 \mathrm{~min}$. followed by filtration through a short plug of Celite ${ }^{\circledR}$ and washing with EtOAc $(200 \mathrm{~mL})$. The filtrate was evaporated in vacuo and the residue was purified by $\mathrm{CC}$. Fractions containing the product were collected and volatile components evaporated in vacuo to give 15a,b and 26c. Compound $\mathbf{1 5 b}$ was isolated as dihydrochloride in the following way. The partially purified free amine obtained by $\mathrm{CC}$ was dissolved in $\mathrm{CH}_{2} \mathrm{Cl}_{2}$ (5 $\mathrm{mL})$ and, while vigorous stirring at r.t., $2 \mathrm{M} \mathrm{HCl}-\mathrm{EtOAc}(2 \mathrm{~mL}, 4 \mathrm{mmol})$ was added. The precipitate was collected by filtration, washed with EtOAc, dried in vacuo to give the dihydrochloride of $\mathbf{1 5 b}$.

3-(2-(Benzyl(methyl)amino)ethyl)-5-hydroxy-1-phenyl-1H-pyrazole (15a). Prepared from 24a (1.605 g, 5 mmol), CC (MeOH/EtOAc, 1:7). Yellowish-brown semi-solid, yield 50\%, 771 mg, IR ( $\left.v_{\max }, \mathrm{cm}^{-1}\right): 3030,2951,2790,1598,1564,1498,1454,1362,1153,1069,1023,907$, 757, 698. ${ }^{1} \mathrm{H}$ NMR (300 MHz, $\left.\mathrm{CDCl}_{3}\right), \delta_{\mathrm{H}} 2.26(3 \mathrm{H}, \mathrm{s}, \mathrm{NMe}), 2.68\left(4 \mathrm{H}, \mathrm{s}, 2 \times \mathrm{CH}_{2}\right), 3.38(2 \mathrm{H}, \mathrm{s}$, $\left.\mathrm{CH}_{2}\right), 3.52\left(2 \mathrm{H}, \mathrm{s}, \mathrm{CH}_{2}\right), 7.14-7.43(8 \mathrm{H}, \mathrm{m}, 8 \mathrm{H}$ of $\mathrm{Ph}), 7.86\left(2 \mathrm{H}, \mathrm{d},{ }^{3} J_{\mathrm{HH}}=8.0 \mathrm{~Hz}, 2 \mathrm{H}\right.$ of $\left.\mathrm{Ph}\right)$. MS, $m / z=308\left(\mathrm{MH}^{+}\right)$, HRMS (ESI), $m / z=308.1759\left(\mathrm{MH}^{+}\right), \mathrm{C}_{19} \mathrm{H}_{22} \mathrm{~N}_{3} \mathrm{O}$ requires 308.1763. Anal. Calcd for $\mathrm{C}_{19} \mathrm{H}_{21} \mathrm{~N}_{3} \mathrm{O}_{3}$ (307.39): C, 74.24; H, 6.89; N, 13.67\%, Found: C, 73.19; H, 6.88; $\mathrm{N}, 13.31 \%$.

5-Hydroxy-1-(4-methoxyphenyl)-3-(2-(pyrrolidin-1-yl)ethyl)-1H-pyrazole dihydrochloride (15b). Prepared from 25b (1.506 g, $5 \mathrm{mmol}$ ), $\mathrm{CC}$ (neutral $\mathrm{Al}_{2} \mathrm{O}_{3}, \mathrm{MeOH} / \mathrm{CH}_{2} \mathrm{Cl}_{2}, 1: 9$ ). White solid, yield 54\%, $981 \mathrm{mg}, \mathrm{mp} 199-201{ }^{\circ} \mathrm{C}$, IR $\left(v_{\max }, \mathrm{cm}^{-1}\right): 3424,2958,2841,2702,2605,2514$, 2359, 2331, 1592, 1580, 1559, 1535, 1508, 1448, 1420, 1400, 1362, 1303, 1257, 1184, 1172, 1109, 1065, 1034, 1018, 850, 799. ' $\mathrm{H}$ NMR (300 MHz, DMSO-d6), $\delta_{\mathrm{H}} 1.80-2.09$ (4H, m, 3"$\mathrm{CH}_{2}, 4$ "- $\left.\mathrm{CH}_{2}\right), 2.88-2.98$ and 2.99-3.10 (4H, 2m, 1:1, $\left.\mathrm{CH}_{2} \mathrm{CH}_{2}\right), 3.35-3.46$ and 3.47-3.60 (4H, 2m, 1:1, 2"- $\mathrm{CH}_{2}, 5$ "- $\left.\mathrm{CH}_{2}\right), 3.78(3 \mathrm{H}, \mathrm{s}, \mathrm{OMe}), 5.52(1 \mathrm{H}, \mathrm{s}, 4-\mathrm{H}$ of pyrazole), 5.93 (2H, br s, $\left.\mathrm{NH}_{2}{ }^{+}\right), 6.96-7.04$ and $7.52-7.61\left(4 \mathrm{H}, 2 \mathrm{~m}, 1: 1, \mathrm{C}_{6} \mathrm{H}_{4}\right), 10.75\left(1 \mathrm{H}\right.$, br s, OH). ${ }^{13} \mathrm{C} \mathrm{NMR}(126 \mathrm{MHz}$, DMSO- $\left.d_{6}\right), \delta_{\mathrm{C}} 22.8,24.2,52.3,52.8,55.5,88.1,114.2,124.0,129.9,146.8,154.0,157.9$. MS, $m / z=288\left(\mathrm{MH}^{+}\right)$, HRMS $(\mathrm{ESI}), m / z=288.1720\left(\mathrm{MH}^{+}\right), \mathrm{C}_{16} \mathrm{H}_{22} \mathrm{~N}_{3} \mathrm{O}_{2}$ requires 288.1712. Anal. Calcd for $\mathrm{C}_{16} \mathrm{H}_{23} \mathrm{Cl}_{2} \mathrm{~N}_{3} \mathrm{O}_{2}$ (360.28): C, 53.34; H, 6.43; N, 11.66\%, Found: C, 52.76; H, 6.41; N, $11.60 \%$.

3-(2-(Dibenzylamino)ethyl)-1-phenyl-1H-pyrazol-5(4H)-one (26c). Prepared from 25c (1.985 $\mathrm{g}, 5 \mathrm{mmol}), \mathrm{CC}$ (EtOAc/hexanes, 1:1). Brownish oil, yield 50\%, $966 \mathrm{mg}$, IR ( $\left.v_{\max }, \mathrm{cm}^{-1}\right): 3060$, 3027, 2928, 2800, 2359, 2341, 1714 (C=O), 1597, 1558, 1497, 1453, 1409, 1365, 1337, 1248, 1154, 1128, 1071, 1027, 977, 906, 749, 697. ${ }^{1} \mathrm{H}$ NMR (300 MHz, $\left.\mathrm{CDCl}_{3}\right), \delta_{\mathrm{H}} 2.61-2.67$ and 2.69-2.76 (4H, 2m, 1:1, $\left.\mathrm{CH}_{2} \mathrm{CH}_{2}\right), 2.99\left(2 \mathrm{H}, \mathrm{s}, 4-\mathrm{CH}_{2}\right.$ of pyrazole), $3.57\left(4 \mathrm{H}, \mathrm{s}, 2 \times \mathrm{CH}_{2} \mathrm{Ph}\right)$, 7.14-7.43 (13H, m, 13H of Ph), 7.84-7.91 (2H, m, 2H of Ph). ${ }^{1} \mathrm{H}$ NMR (500 MHz, DMSO- $\left.d_{6}\right)$, 
$\delta_{\mathrm{H}} 3.07-3.13$ and 3.19-3.25 (4H, 2m, 1:1, $\left.\mathrm{CH}_{2} \mathrm{CH}_{2} \mathrm{NBn}_{2}\right), 4.39\left(4 \mathrm{H}, \mathrm{s}, 2 \times \mathrm{CH}_{2} \mathrm{Ph}\right), 5.44(1 \mathrm{H}, \mathrm{s}, 4-$ $\mathrm{H}$ of pyrazole), $7.24\left(1 \mathrm{H}, \mathrm{t},{ }^{3} J_{\mathrm{HH}}=7.4 \mathrm{~Hz}, 1 \mathrm{H}\right.$ of $\left.\mathrm{Ph}\right), 7.41-7.45(9 \mathrm{H}, \mathrm{m}, 9 \mathrm{H}$ of $\mathrm{Ph}), 7.62-7.65$ $(2 \mathrm{H}, \mathrm{m}, 2 \mathrm{H}$ of $\mathrm{Ph}), 7.69-7.73(3 \mathrm{H}, \mathrm{m}, 3 \mathrm{H}$ of $\mathrm{Ph}), 11.55\left(1 \mathrm{H}\right.$, br s, OH). ${ }^{13} \mathrm{C} \mathrm{NMR}(75.5 \mathrm{MHz}$, DMSO- $\left.d_{6}\right), \delta_{\mathrm{C}} 22.7,22.7,50.0,56.1,87.4,121.1,121.1,125.8,128.6,128.8,128.9$, 129.0, 129.5, 130.1, 130.3, 131.5, 138.2, 147.6, 153.8. MS, $m / z=384\left(\mathrm{MH}^{+}\right), \mathrm{HRMS}(\mathrm{ESI}), \mathrm{m} / z=$ 384.2080 $\left(\mathrm{MH}^{+}\right), \mathrm{C}_{25} \mathrm{H}_{25} \mathrm{~N}_{3} \mathrm{O}$ requires 384.2076. Anal. Calcd for $\mathrm{C}_{25} \mathrm{H}_{25} \mathrm{~N}_{3} \mathrm{O}$ (383.49): C, 78.30; H, 6.57; N, 10.96\%, Found: C, 79.59; H, 6.74; N, 10.83\%.

\section{Acknowledgements}

We acknowledge with thanks the financial support from Boehringer-Ingelheim Pharma GmbH \& Co. KG (Biberach, Germany). The financial support from the Slovenian Research Agency through grant P1-0179 is gratefully acknowledged.

\section{References}

1. (a) Patrick, G. L. An Introduction to Medicinal Chemistry; $3^{\text {rd }}$ Edn., Oxford University Press: Oxford, 2005. (b) Kazuta, Y.; Hirano, K.; Natsume, K.; Yamada, S.; Kimura, R.; Matsumoto, S.-i.; Furuichi, K.; Matsuda, A.; Shuto, S. J. Med. Chem. 2003, 46, 1980. (c) Paillet-Loilier, M.; Fabis, F.; Lepailleur, A.; Bureau, R.; Butt-Gueulle, S.; Dauphin, F.; Lesnard, A.; Delarue, C.; Vaudryb, H.; Rault, S. Bioorg. Med. Chem. Lett. 2007, 17, 3018. (d) Pullagurla, M.; Dukat, M.; Roth, B. L.; Setola, V.; Glennon, R. A. Med. Chem. Res. 2005, 14, 1. (e) Liebscher, J.; Patzel, M. Synlett 1994, 471.

2. (a) Yet, L. Pyrazoles In Comprehensive Heterocyclic Chemistry III; Katritzky, A. R.; Ramsden, C. A.; Scriven, E. F. V.; Taylor, R. J. K., Eds., Vol. 4, Joule, J. A., Ed., Elsevier: Oxford, 2008, pp. 1-141. (b) Stanovnik, B.; Svete, J. Pyrazoles In Science of Synthesis, Houben-Weyl Methods of Organic Transformations; Neier, R. Ed., Vol. 12, Georg Thieme Verlag, Stuttgart, 2002, pp. 15-225. (c) Stanovnik, B.; Grošelj, U. Adv. Heterocycl. Chem. 2010, 100, 145.

3. (a) N. Xi, Q. Huang, L. Lin, Imidazoles In Comprehensive Heterocyclic Chemistry III; Katritzky, A. R.; Ramsden, C. A.; Scriven, E. F. V.; Taylor, R. J. K., Vol. 4, J. Joule, Ed., Elsevier: Oxford, 2008, pp. 143-364. (b) Grimmett, M. R Imidazoles In Science of Synthesis, Houben-Weyl Methods of Organic Transformations; Neier, R., Ed., Vol. 12, Georg Thieme Verlag, Stuttgart, 2002, pp. 325-528.

4. (a) Kralj, D.; Grošelj, U. Meden, A.; Dahmann, G.; Stanovnik, B.; Svete, J. Tetrahedron 2007, 63, 11213. (b) Kralj, D.; Novak, A.; Dahmann, G.; Grošelj, U.; Meden, A.; Svete, J. J. Comb. Chem. 2008, 10, 664. 
5. Kralj, D.; Friedrich, M.; Grošelj, U.; Kiraly-Potpara, S.; Meden, A.; Wagger, J.; Dahmann, G.; Stanovnik, B.; Svete, J. Tetrahedron 2009, 65, 7151.

6. Žerovnik, D.; Grošelj, U.; Kralj, D.; Malavašič, Č.; Bezenšek, J.; Dahmann, G.; Stare, K.; Meden, A.; Stanovnik, B.; Svete, J. Synthesis 2010, 3633.

7. Janjić, M.; Prebil, R.; Grošelj, U.; Kralj, D.; Malavašič, Č.; Golobič, A.; Stare, K.; Dahmann, G.; Stanovnik, B.; Svete, J. Helv. Chim. Acta 2011, 94, 1703.

8. (a) Brooks, D. W.; Lu, L. D.-L.; Masamune, S. Angew. Chem. Int. Ed. 1979, 18, 72. (b) Hashiguchi, S.; Kawada, A.; Natsugari, H.Synthesis 1992, 403. (c) Moreau, R. J.; Sorensen, E. J. Tetrahedron 2007, 63, 6446.

9. (a) S. J. Miller and D. J. Guerin in Enantioselective Synthesis of $\beta$-Amino Acids, $2^{\text {nd }}$ Edn, John Wiley \& Sons, Inc.: 2005, Hoboken, N. J., pp. 351-376. (b) Liu M.; Sibi, M. P. In Enantioselective Synthesis of $\beta$-Amino Acids, $2^{\text {nd }}$ Edn, Wiley: Hoboken, N. J., 2005, pp. 377-395.

10. (a) Yeom, C.-E.; Kim, M. J.; Kim, B. M. Tetrahedron 2007, 63, 904. (b) Le Sann, C.; Huddleston, J.; Mann, J. Tetrahedron 2007, 63, 12903. (c) Ranu, B. C.; Dey, S. S.; Hajra, A. ARKIVOC 2002, (vii), 76.

11. (a) Liu, S.; Yang, Y.-L. A.; Sambandam, A.; Molino, B. F.; Olson, R. E. WO 2008141082; Chem. Abstr. 2008, 149, 576408. (b) Yamada, N.; Sugi, H.; Kon, K. EP 219852; Chem. Abstr. 1990, 112, 139733.

12. (a) D. Bevk, R. Jakše, J. Svete, A. Golobič, L. Golič, and B. Stanovnik, Heterocycles 2003, 61, 197. (b) A. P. Yakubov, D. V. Tsyganov, Y. M. Emel'yanova, and V. N. Nesterov, Khim. Geterotsikl. Soed. (Chem. Heterocycl.Compds.) 2002, 38, 1180. (c) K. Fukushi, M. Nakaya, S. Shiraishi, M. Nakamura, and S. Numata, Jpn. Kokai Tokkyo Koho JP 01056690, 1989, Chem. Abstr. 1989, 111, 134146. (d) Jpn. Kokai Tokkyo Koho JP 82 40,467, 1982, Chem. Abstr. 1982, 97, 92271. (e) Fujimura, H.; Hori, M.; Kojima, S.; Kato, K.; Sugiura, S. Jpn. Kokai Tokkyo Koho JP49011232, 1974, Chem. Abstr. 1974, 81, 136138. (f) Short, F. W.; Schoeb, E. J. J. Heterocycl. Chem. 1969, 6, 723. (g) von Pechmann, H. Liebigs Ann. Chem. 1891, 261, 171.

13. (a) Holzer, W.; Kautsch, C.; Laggner, C.; Claramunt, R. M.; Perez-Torralba, M.; Alkorta, I.; Elguero, J. Tetrahedron 2004, 60, 6791. (b) Katritzky, A. R.; Karelson, M.; Harris, P. A.; Heterocycles 1991, 32, 329. (c) Dorn, H. J. Prakt. Chem. 1973, 315, 382.

14. In the case of hydrazine hydrochloride, one equivalent of $\mathrm{Et}_{3} \mathrm{~N}(0.14 \mathrm{~mL}, 1 \mathrm{mmol})$ was added as well. 